\title{
A GEOMETRIC CONSTRUCTION OF CRYSTAL GRAPHS USING QUIVER VARIETIES: EXTENSION TO THE NON-SIMPLY LACED CASE
}

\author{
ALISTAIR SAVAGE
}

\begin{abstract}
We consider a generalization of the quiver varieties of Lusztig and Nakajima to the case of all symmetrizable Kac-Moody Lie algebras. To deal with the non-simply laced case one considers admissible automorphisms of a quiver and the irreducible components of the quiver varieties fixed by this automorphism. We define a crystal structure on these irreducible components and show that the crystals obtained are isomorphic to those associated to the crystal bases of the lower half of the universal enveloping algebra and the irreducible highest weight representations of the non-simply laced Kac-Moody Lie algebra. As an application, we realize the crystal of the spin representation of $\mathfrak{s o}_{2 n+1}$ on the set of self-conjugate Young diagrams that fit inside an $n \times n$ box.
\end{abstract}

\section{INTRODUCTION}

In [10], Lusztig associated various varieties to the quiver (oriented graph) whose underlying graph is the Dynkin graph of a simply laced Kac-Moody algebra (that is, a Kac-Moody algebra with symmetric Cartan matrix). Using these quiver varieties, he gave a geometric realization of $U_{q}^{-}(\mathfrak{g})$, the lower half of the quantum universal enveloping algebra of $\mathfrak{g}$, and defined a canonical basis [9] with remarkable properties. Later, Nakajima extended this quiver variety construction to yield a geometric realization of the entire universal enveloping algebra and its irreducible highest weight representations in the homology of a new class of quiver varieties. This geometric approach allows one to prove such results as positivity and integrality of the canonical basis that are difficult to prove algebraically.

In [6], Kashiwara constructed the crystal base and the global crystal base of $U_{q}^{-}(\mathfrak{g})$ and the highest weight representations of $U_{q}(\mathfrak{g})$ in an algebraic way. It was shown by Grojnowski and Lusztig [2] that the global crystal base and the canonical basis coincide. Kashiwara and Saito [7, 16] defined a crystal structure on the set of irreducible components of Lusztig's and Nakajima's quiver varieties and showed that the crystal obtained is isomorphic to that associated to the crystal base of the lower half of the universal enveloping algebra and the irreducible highest weight representation respectively. There are other, purely combinatorial, constructions of these crystals as well. In [17], the author described an explicit isomorphism between the geometric construction from quiver varieties and the combinatorial construction via Young tableaux and Young walls.

Date: June 3, 2004.

2000 Mathematics Subject Classification. 17B37,16G20.

This research was supported by the Natural Sciences and Engineering Research Council (NSERC) of Canada. 
One limitation of the quiver variety approach to Kac-Moody Lie algebras and their representations is that the geometric Lie algebra action is only defined for those algebras whose generalized Cartan matrix is symmetric. Thus, even for the finite case, only types $\mathrm{A}, \mathrm{D}$, and $\mathrm{E}$ are covered. However, the other algebraic structures mentioned above, such as global bases and crystal graphs, are defined for the more general case of symmetrizable Kac-Moody Lie algebras. In the current paper, we consider an extension of the geometric construction of crystal bases to this more general setting. We consider quiver varieties for symmetrizable KacMoody Lie algebras (which are not necessarily simply laced) and introduce a crystal structure on their irreducible components. In particular, we can treat all of the finite dimensional simple Lie algebras. We give here a brief overview of the construction.

Associated to a symmetrizable Kac-Moody Lie algebra (or its generalized Cartan matrix), one can associate a valued graph $\Gamma$. Let $\mathfrak{g}(\Gamma)$ denote the Kac-Moody Lie algebra. It is known [11, Prop 14.1.2] that any such valued graph can be obtained from a quiver $Q$ along with an admissible automorphism a of this graph. This admissible automorphism induces an automorphism on the set of irreducible components of the quiver varieties associated to $Q$. Let $\mathbf{w}$ be a dominant integral weight of $\mathfrak{g}(Q)$, the Kac-Moody Lie algebra with Dynkin diagram the underlying graph of $Q$, which is fixed by the automorphism of the weight lattice induced by $\mathbf{a}$. Then let $B_{Q}^{g}(\infty)$ and $B_{Q}^{g}(\mathbf{w})$ be the set of irreducible components of Lusztig's and Nakajima's quiver varieties attached to the quiver $Q$ and let $B_{\Gamma}^{g}(\infty)$ and $B_{\Gamma}^{g}(\mathbf{w})$ be the subsets that are fixed by the automorphism $\mathbf{a}$. Let $\mathbf{I}$ be the set of $\mathbf{a}$-orbits of vertices of $Q$. It turns out that for $\mathbf{i} \in \mathbf{I}$ and $i, j \in \mathbf{i}$, the Kashiwara operators $\tilde{e}_{i}$ and $\tilde{e}_{j}$ (resp. $\tilde{f}_{i}$ and $\tilde{f}_{j}$ ) which act on the sets $B_{Q}^{g}(\infty)$ and $B_{Q}^{g}(\mathbf{w})$ commute and the operators $\tilde{e}_{\mathbf{i}}=\prod_{i \in \mathbf{i}} \tilde{e}_{i}$ and $\tilde{f}_{\mathbf{i}}=\prod_{i \in \mathbf{i}} \tilde{f}_{i}$ act on the sets $B_{\Gamma}^{g}(\infty)$ and $B_{\Gamma}^{g}(\mathbf{w})$. These operators (along with the other maps required to define a crystal structure) endow these sets with the structure of the crystal associated to the crystal base of $U_{q}^{-}(\mathfrak{g}(\Gamma))$ and $V_{\Gamma}(\mathbf{w})$, the irreducible highest weight representation of $U_{q}(\mathfrak{g}(\Gamma))$ of highest weight $\mathbf{w}$. We can also define these operators directly, in geometric terms, without reference to the operators $\tilde{e}_{i}$ and $\tilde{f}_{i}$ for the quiver $Q$.

As an application of our construction, we describe an explicit realization of the crystal graph of the spin representation of $\mathfrak{s o}_{2 n+1}$ on the set of self-conjugate Young diagrams that fit inside an $n \times n$ box. Each Young diagram corresponds to an irreducible component of the corresponding quiver variety. It turns out that we can actually extend this crystal action to the full action of the Lie algebra and realize the spin representation on the vector space spanned by these Young diagrams. This is similar to the realization of the spin representations of $\mathfrak{s o}_{2 n}$ obtained in [18].

The fact that the invariant irreducible components $B_{\Gamma}^{g}(\mathbf{w})$ of Nakajima's quiver varieties enumerate a basis for highest weight representations of the corresponding representation of the non-simply laced Kac-Moody Lie algebra was demonstrated by F. Xu [19]. The proof in [19] uses perverse sheaves while our method avoids their use and is almost entirely crystal theoretic. The crystal structure on $B_{\Gamma}^{g}(\infty)$ was mentioned in [19] but not defined. A key step in our construction is the embedding of crystals of non-simply laced type into those of simply laced type. We should note that this has been done in some specific cases for Lie algebras of affine type (see, for example, $[14,15])$. Our method works for arbitrary symmetrizable Kac-Moody algebras. 
If we define the quiver variety associated to the valued graph $\Gamma$ to be the union of the irreducible components of Lusztig's (resp. Nakajima's) quiver variety associated to $Q$ that are fixed by the isomorphism a, then by the above we know that the irreducible components of this new quiver variety are in 1-1 correspondence with a basis for $U_{q}^{-}\left(\mathfrak{g}(\Gamma)\right.$ ) (resp. $V_{\Gamma}(\mathbf{w})$ ). Thus, the top dimensional Borel-Moore homology of these quiver varieties has the same dimension (in each weight space) as $U_{q}^{-}(\mathfrak{g}(\Gamma))$ (resp. $V_{\Gamma}(\mathbf{w})$ ). Therefore, one should be able to define the action of $U_{q}(\mathfrak{g}(\Gamma))$ on these spaces via convolution in homology and obtain a geometric realization of $U_{q}(\mathfrak{g}(\Gamma))$ and $V_{\Gamma}(\mathbf{w})$ as in [13].

One immediate application of the current work is an extension of the results of [17]. The combinatorial methods of Young tableaux and Young walls exist for nonsimply laced Kac-Moody algebras and thus the connection between the geometric and combinatorial realizations of the crystal graphs should be able to be extended to the more general setting. Ideally one should be able to describe an explicit isomorphism between the geometric realization and the path model of Littelmann [8] which exists in the full generality of symmetrizable Kac-Moody Lie algebras.

The organization of the paper is as follows. In Sections 1 and 2 we review the definitions of Lusztig's and Nakajima's quiver varieties and in Sections 3 and 4 we recall the crystal action on their irreducible components. In Section 5 we prove a result about realizing the crystal of $U_{q}^{-}(\mathfrak{g}(\Gamma))$ inside the crystal of $U_{q}^{-}(\mathfrak{g}(Q))$. We describe the geometric realization of the crystal of $U_{q}^{-}(\mathfrak{g}(\Gamma))$ in Section 6. In Section 7 we show how to realize the crystal of $V_{\Gamma}(\mathbf{w})$ inside that for $V_{Q}(\mathbf{w})$ and in Section 8 we develop the geometric realization of the crystal of $V_{\Gamma}(\mathbf{w})$. Finally, in Section 9 we realize the crystal graph of the spin representation of $\mathfrak{s o}_{2 n+1}$.

The author would like to thank I.B. Frenkel and C.M. Ringel for useful discussions and suggestions. He would also like to thank H. Nakajima for bringing the reference [19] to his attention.

\section{LUSZTIG'S QUIVER VARIETY}

In this section, we will recount the description given in [10] of Lusztig's quiver varieties. See this reference for details, including proofs.

Let $I$ and $E$ be the set of vertices and (unoriented) edges of the Dynkin graph of a Kac-Moody Lie algebra with symmetric Cartan matrix and let $H$ be the set of pairs consisting of an edge together with an orientation of it. We denote the corresponding quiver by $Q=(I, H)$ and the Kac-Moody Lie algebra by $\mathfrak{g}(Q)$. For $h \in H$, let $\operatorname{in}(h)$ (resp. out $(h)$ ) be the incoming (resp. outgoing) vertex of $h$. We define the involution ${ }^{-}: H \rightarrow H$ to be the function which takes $h \in H$ to the element of $H$ consisting of the same edge with opposite orientation. An orientation of our graph is a choice of a subset $\Omega \subset H$ such that $\Omega \cup \bar{\Omega}=H$ and $\Omega \cap \bar{\Omega}=\emptyset$.

Let $\mathcal{V}$ be the category of finite-dimensional $I$-graded vector spaces $\mathbf{V}=\oplus_{i \in I} \mathbf{V}_{i}$ over $\mathbb{C}$ with morphisms being linear maps respecting the grading. Then $\mathbf{V} \in \mathcal{V}$ shall denote that $\mathbf{V}$ is an object of $\mathcal{V}$. We identify the graded dimension $\mathbf{v}$ of $\mathbf{V}$ with the element $\sum_{i \in I} \mathbf{v}_{i} \alpha_{i}$ of the root lattice of $\mathfrak{g}(Q)$. Here the $\alpha_{i}$ are the simple roots corresponding to the vertices of our quiver.

Given $\mathbf{V} \in \mathcal{V}$, let

$$
\mathbf{E}(\mathbf{v})=\bigoplus_{h \in H} \operatorname{Hom}\left(\mathbf{V}_{\mathrm{out}(h)}, \mathbf{V}_{\mathrm{in}(h)}\right)
$$


Note that this space (and the others that follow) depend only on the dimension of $\mathbf{V}$ up to isomorphism. This is why we label the space by the dimension $\mathbf{v}$. For any subset $H^{\prime} \subset H$, let $\mathbf{E}_{H^{\prime}}(\mathbf{v})$ be the subspace of $\mathbf{E}(\mathbf{v})$ consisting of all vectors $x=\left(x_{h}\right)$ such that $x_{h}=0$ whenever $h \notin H^{\prime}$. The algebraic group $G_{\mathbf{v}}=\prod_{i} \operatorname{Aut}\left(\mathbf{V}_{i}\right)$ acts on $\mathbf{E}(\mathbf{v})$ and $\mathbf{E}_{H^{\prime}}(\mathbf{v})$ by

$$
(g, x)=\left(\left(g_{i}\right),\left(x_{h}\right)\right) \mapsto\left(g_{\mathrm{in}(h)} x_{h} g_{\text {out }(h)}^{-1}\right) .
$$

Define the function $\varepsilon: H \rightarrow\{-1,1\}$ by $\varepsilon(h)=1$ for all $h \in \Omega$ and $\varepsilon(h)=-1$ for all $h \in \bar{\Omega}$. The Lie algebra of $G_{\mathbf{v}}$ is $\mathbf{g l}_{\mathbf{v}}=\prod_{i} \operatorname{End}\left(\mathbf{V}_{i}\right)$ and it acts on $\mathbf{E}(\mathbf{v})$ by

$$
(a, x)=\left(\left(a_{i}\right),\left(x_{h}\right)\right) \mapsto[a, x]=\left(x_{h}^{\prime}\right)=\left(a_{\operatorname{in}(h)} x_{h}-x_{h} a_{\text {out }(h)}\right) .
$$

Let $\langle\cdot, \cdot\rangle$ be the nondegenerate, $G_{\mathbf{v}}$-invariant, symplectic form on $\mathbf{E}(\mathbf{v})$ with values in $\mathbb{C}$ defined by

$$
\langle x, y\rangle=\sum_{h \in H} \varepsilon(h) \operatorname{tr}\left(x_{h} y_{\bar{h}}\right) .
$$

Note that $\mathbf{E}(\mathbf{v})$ can be considered as the cotangent space of $\mathbf{E}_{\Omega}(\mathbf{v})$ under this form.

The moment map associated to the $G_{\mathbf{v}}$-action on the symplectic vector space $\mathbf{E}(\mathbf{v})$ is the map $\psi: \mathbf{E}(\mathbf{v}) \rightarrow \mathbf{g l}_{\mathbf{v}}$ with $i$-component $\psi_{i}: \mathbf{E}(\mathbf{v}) \rightarrow$ End $\mathbf{V}_{i}$ given by

$$
\psi_{i}(x)=\sum_{h \in H, \operatorname{in}(h)=i} \varepsilon(h) x_{h} x_{\bar{h}} .
$$

Definition $1.1([10])$. An element $x \in \mathbf{E}(\mathbf{v})$ is said to be nilpotent if there exists an $N \geq 1$ such that for any sequence $h_{1}, h_{2}, \ldots, h_{N}$ in $H$ satisfying out $\left(h_{1}\right)=\operatorname{in}\left(h_{2}\right)$, $\operatorname{out}\left(h_{2}\right)=\operatorname{in}\left(h_{3}\right), \ldots, \operatorname{out}\left(h_{N-1}\right)=\operatorname{in}\left(h_{N}\right)$, the composition $x_{h_{1}} x_{h_{2}} \ldots x_{h_{N}}$ : $\mathbf{V}_{\text {out }\left(h_{N}\right)} \rightarrow \mathbf{V}_{\text {in }\left(h_{1}\right)}$ is zero.

Definition $1.2([10])$. Let $\mathbf{E}^{0}(\mathbf{v})$ be the set of elements $x \in \mathbf{E}(\mathbf{v})$ such that $\psi_{i}(x)=$ 0 for all $i \in I$. Let $\Lambda(\mathbf{v})$ be the subset of $\mathbf{E}^{0}(\mathbf{v})$ consisting of nilpotent elements.

Proposition $1.3([10])$. For $\mathfrak{g}(Q)$ a Kac-Moody Lie algebra of finite type with symmetric Cartan matrix, the irreducible components of $\Lambda(\mathbf{v})$ are the closures of the conormal bundles of the various $G_{\mathbf{v}}$-orbits in $\mathbf{E}_{\Omega}(\mathbf{v})$.

\section{NAKAJIMA'S QUIVER VARIETY}

We introduce here a description of the quiver varieties first presented in [12]. See [12] and [13] for details.

Definition $2.1([12])$. For $\mathbf{v}, \mathbf{w} \in\left(\mathbb{Z}_{>0}\right)^{I}$, choose I-graded vector spaces $\mathbf{V}$ and $\mathbf{W}$ of graded dimensions $\mathbf{v}$ and $\mathbf{w}$ respectively. We associate $\mathbf{w}$ with the element $\sum_{i} \mathbf{w}_{i} \omega_{i}$ of the root lattice of $\mathfrak{g}(Q)$, where the $\omega_{i}$ are the fundamental weights of $\mathfrak{g}(Q)$. Recall that we identified $\mathbf{v}$ with the weight $\sum_{i} \mathbf{v}_{i} \alpha_{i}$. Then define

$$
\Lambda \equiv \Lambda(\mathbf{v} ; \mathbf{w})=\Lambda(\mathbf{v}) \times \bigoplus_{i \in I} \operatorname{Hom}\left(\mathbf{V}_{i}, \mathbf{W}_{i}\right)
$$

Now, suppose that $\mathbf{S}$ is an $I$-graded subspace of $\mathbf{V}$. For $x \in \Lambda(\mathbf{v})$ we say that $\mathbf{S}$ is $x$-stable if $x(\mathbf{S}) \subset \mathbf{S}$.

Definition $2.2([12])$. Let $\Lambda^{\text {st }}=\Lambda(\mathbf{v} ; \mathbf{w})^{\text {st }}$ be the set of all $(x, t) \in \Lambda(\mathbf{v} ; \mathbf{w})$ satisfying the following condition: If $\mathbf{S}=\left(\mathbf{S}_{i}\right)$ with $\mathbf{S}_{i} \subset \mathbf{V}_{i}$ is x-stable and $t_{i}\left(\mathbf{S}_{i}\right)=0$ for all $i \in I$, then $\mathbf{S}_{i}=0$ for all $i \in I$. 
The group $G_{\mathbf{v}}$ acts on $\Lambda(\mathbf{v} ; \mathbf{w})$ via

$$
(g,(x, t))=\left(\left(g_{i}\right),\left(\left(x_{h}\right),\left(t_{i}\right)\right)\right) \mapsto\left(\left(g_{\mathrm{in}(h)} x_{h} g_{\text {out }(h)}^{-1}\right),\left(t_{i} g_{i}^{-1}\right)\right) .
$$

and the stabilizer of any point of $\Lambda(\mathbf{v} ; \mathbf{w})^{\text {st }}$ in $G_{\mathbf{v}}$ is trivial (see [13, Lemma 3.10]). We then make the following definition.

Definition $2.3([12])$. Let $\mathcal{L} \equiv \mathcal{L}(\mathbf{v}, \mathbf{w})=\Lambda(\mathbf{v} ; \mathbf{w})^{s t} / G_{\mathbf{v}}$.

\section{Crystal action on Lusztig's Quiver varieties}

In this section we recall the crystal action on the set of irreducible components of Lusztig's quiver varieties. See [7] for details, including proofs.

Let $\mathbf{v}, \overline{\mathbf{v}}, \mathbf{v}^{\prime} \in\left(\mathbb{Z}_{\geq 0}\right)^{I}$ such that $\mathbf{v}=\overline{\mathbf{v}}+\mathbf{v}^{\prime}$ and let $\mathbf{V}, \overline{\mathbf{V}}, \mathbf{V}^{\prime}$ have dimensions $\mathbf{v}, \overline{\mathbf{v}}, \mathbf{v}^{\prime}$ respectively. Consider the maps

$$
\mathbf{E}^{0}(\overline{\mathbf{v}}) \times \mathbf{E}^{0}\left(\mathbf{v}^{\prime}\right) \stackrel{q_{1}}{\longleftarrow} \mathbf{E}^{0}\left(\overline{\mathbf{v}}, \mathbf{v}^{\prime}\right) \stackrel{q_{2}}{\longrightarrow} \mathbf{E}^{0}(\mathbf{v}),
$$

where $\mathbf{E}^{0}\left(\overline{\mathbf{v}}, \mathbf{v}^{\prime}\right)$ is the variety of $\left(x, \bar{\phi}, \phi^{\prime}\right)$, where $x \in \mathbf{E}^{0}(\mathbf{v})$ and $\bar{\phi}=\left(\bar{\phi}_{i}\right), \phi^{\prime}=\left(\phi_{i}^{\prime}\right)$ give an exact sequence

$$
0 \longrightarrow \overline{\mathbf{V}}_{i} \stackrel{\bar{\phi}_{i}}{\longrightarrow} \mathbf{V}_{i} \stackrel{\phi_{i}^{\prime}}{\longrightarrow} \mathbf{V}_{i}^{\prime} \longrightarrow 0
$$

such that $\operatorname{Im} \bar{\phi}$ is stable under $x$. Thus $x$ induces $\bar{x}: \overline{\mathbf{V}} \rightarrow \overline{\mathbf{V}}$ and $x^{\prime}: \mathbf{V}^{\prime} \rightarrow \mathbf{V}^{\prime}$. The maps $q_{1}$ and $q_{2}$ are defined by $q_{1}\left(x, \bar{\phi}, \phi^{\prime}\right)=\left(\bar{x}, x^{\prime}\right)$ and $q_{2}\left(x, \bar{\phi}, \phi^{\prime}\right)=x$.

Lemma 3.1 ([7]). The following conditions are equivalent.

(1) $x$ is nilpotent

(2) Both $\bar{x}$ and $x^{\prime}$ are nilpotent.

Thus (3.1) induces the maps

$$
\Lambda(\overline{\mathbf{v}}) \times \Lambda\left(\mathbf{v}^{\prime}\right) \stackrel{q_{1}}{\longleftarrow} \Lambda^{\prime}\left(\overline{\mathbf{v}}, \mathbf{v}^{\prime}\right) \stackrel{q_{2}}{\longrightarrow} \Lambda(\mathbf{v}),
$$

where $\Lambda^{\prime}\left(\overline{\mathbf{v}}, \mathbf{v}^{\prime}\right)=q_{2}^{-1}(\Lambda(\mathbf{v}))=q_{1}^{-1}\left(\Lambda(\overline{\mathbf{v}}) \times \Lambda\left(\mathbf{v}^{\prime}\right)\right)$.

For $i \in I$ and $p \in \mathbb{Z}_{\geq 0}$, let

$$
\varepsilon_{i}(x)=\operatorname{dim} \text { Coker }\left(\bigoplus_{h: \operatorname{in}(h)=i} V_{\text {out }(h)} \stackrel{\left(x_{h}\right)}{\longrightarrow} V_{i}\right),
$$

and

$$
\mathbf{E}^{0}(\mathbf{v})_{i, p}=\left\{x \in \mathbf{E}^{0}(\mathbf{v}) \mid \varepsilon_{i}(x)=p\right\} .
$$

Then $\mathbf{E}^{0}(\mathbf{v})_{i, p}$ is a locally closed subvariety of $\mathbf{E}^{0}(\mathbf{v})$.

Now assume that $\mathbf{v}=\overline{\mathbf{v}}+c \alpha_{i}$ for $c \in \mathbb{Z}_{\geq 0}$ and consider (3.1). One easily sees that $\mathbf{E}^{0}\left(c \alpha_{i}\right)=\{0\}$. Thus we have

$$
\mathbf{E}^{0}(\overline{\mathbf{v}}) \cong \mathbf{E}^{0}(\overline{\mathbf{v}}) \times \mathbf{E}^{0}\left(c \alpha_{i}\right) \stackrel{\varpi_{1}}{\longleftarrow} \mathbf{E}^{0}\left(\overline{\mathbf{v}}, c \alpha_{i}\right) \stackrel{\varpi_{2}}{\longrightarrow} \mathbf{E}^{0}(\mathbf{v}) .
$$

For $p \in \mathbb{Z}_{\geq 0}$ we have

$$
\varpi_{1}^{-1}\left(\mathbf{E}^{0}(\overline{\mathbf{v}})_{i, p}\right)=\varpi_{2}^{-1}\left(\mathbf{E}^{0}(\mathbf{v})_{i, p+c}\right) .
$$

Thus we define

$$
\mathbf{E}^{0}\left(\overline{\mathbf{v}}, c \alpha_{i}\right)_{i, p}=\varpi_{1}^{-1}\left(\mathbf{E}^{0}(\overline{\mathbf{v}})_{i, p}\right)=\varpi_{2}^{-1}\left(\mathbf{E}^{0}(\mathbf{v})_{i, p+c}\right) .
$$

Setting $p=0$ we have the following diagram

$$
\mathbf{E}^{0}(\overline{\mathbf{v}}) \supset \mathbf{E}^{0}(\overline{\mathbf{v}})_{i, 0} \stackrel{\varpi_{1}}{\longleftarrow} \mathbf{E}^{0}\left(\overline{\mathbf{v}}, c \alpha_{i}\right)_{i, 0} \stackrel{\varpi_{2}}{\longrightarrow} \mathbf{E}^{0}(\mathbf{v})_{i, c} \subset \mathbf{E}^{0}(\mathbf{v}) .
$$


Note that $\mathbf{E}^{0}(\overline{\mathbf{v}})_{i, 0}$ is an open subvariety of $\mathbf{E}^{0}(\overline{\mathbf{v}})$.

Lemma $3.2([7])$. We have the following.

(1) $\varpi_{2}: \mathbf{E}^{0}\left(\overline{\mathbf{v}}, c \alpha_{i}\right)_{i, 0} \rightarrow \mathbf{E}^{0}(\mathbf{v})_{i, c}$ is a principal fiber bundle with $G L\left(\mathbb{C}^{c}\right) \times$ $\prod_{j \in I} G L\left(\overline{\mathbf{V}}_{j}\right)$ as fiber.

(2) $\varpi_{1}: \mathbf{E}^{0}\left(\overline{\mathbf{v}}, c \alpha_{i}\right)_{i, 0} \rightarrow \mathbf{E}^{0}(\overline{\mathbf{v}})_{i, 0}$ is a smooth map whose fiber is a connected rational variety.

Let $B_{Q}^{g}(\mathbf{v}, \infty)$ be the set of irreducible components of $\Lambda(\mathbf{v})$. For $X \in B_{Q}^{g}(\mathbf{v}, \infty)$, we define $\varepsilon_{i}(X)=\varepsilon_{i}(x)$ for a generic point $x$ of $X$. For $p \in \mathbb{Z}_{\geq 0}$, let $B_{Q}^{g}(\mathbf{v}, \infty)_{i, p}$ denote the set of all elements $X$ of $B_{Q}^{g}(\mathbf{v}, \infty)$ such that $\varepsilon_{i}(X)=p$. From the above lemma, we obtain the following.

Proposition 3.3 ([7]). We have

$$
B_{Q}^{g}(\overline{\mathbf{v}} ; \infty)_{i, 0} \cong B_{Q}^{g}(\mathbf{v}, \infty)_{i, c} .
$$

Suppose that $\bar{X} \in B_{Q}^{g}(\overline{\mathbf{v}} ; \infty)_{i, 0}$ corresponds to $X \in B_{Q}^{g}(\mathbf{v}, \infty)_{i, c}$ by the above isomorphism. Then we define

$$
\begin{array}{ll}
\tilde{f}_{i}^{c}: B_{Q}^{g}(\overline{\mathbf{v}} ; \infty)_{i, 0} \rightarrow B_{Q}^{g}(\mathbf{v}, \infty)_{i, c}, & \tilde{f}_{i}^{c}(\bar{X})=X, \\
\tilde{e}_{i}^{c}: B_{Q}^{g}(\mathbf{v}, \infty)_{i, c} \rightarrow B_{Q}^{g}(\overline{\mathbf{v}} ; \infty)_{i, 0}, & \tilde{e}_{i}^{c}(X)=\bar{X} .
\end{array}
$$

We then define maps

$$
\begin{gathered}
\tilde{e}_{i}: \bigsqcup_{\mathbf{v}} B_{Q}^{g}(\mathbf{v}, \infty) \rightarrow \bigsqcup_{\mathbf{v}} B_{Q}^{g}(\mathbf{v}, \infty) \sqcup\{0\}, \\
\tilde{f}_{i}: \bigsqcup_{\mathbf{v}} B_{Q}^{g}(\mathbf{v}, \infty) \rightarrow \bigsqcup_{\mathbf{v}} B_{Q}^{g}(\mathbf{v}, \infty),
\end{gathered}
$$

as follows. For $c>0$, we define

$$
\tilde{e}_{i}: B_{Q}^{g}(\mathbf{v}, \infty)_{i, c} \stackrel{\tilde{e}_{i}^{c}}{\longrightarrow} B_{Q}^{g}(\overline{\mathbf{v}} ; \infty)_{i, 0} \stackrel{\tilde{f}_{i}^{c-1}}{\longrightarrow} B_{Q}^{g}\left(\mathbf{v}-\alpha_{i} ; \infty\right)_{i, c-1},
$$

and $\tilde{e}_{i}(X)=0$ for $X \in B_{Q}^{g}(\mathbf{v}, \infty)_{i, 0}$. We define

$$
\tilde{f}_{i}: B_{Q}^{g}(\mathbf{v}, \infty)_{i, c} \stackrel{\tilde{e}_{i}^{c}}{\longrightarrow} B_{Q}^{g}(\infty ; \overline{\mathbf{v}})_{i, 0} \stackrel{\tilde{f}_{i}^{c+1}}{\longrightarrow} B_{Q}^{g}\left(\infty ; \mathbf{v}+\alpha_{i}\right)_{i, c+1} .
$$

Furthermore, we define a map

$$
\text { wt }: \bigsqcup_{\mathbf{v}} B_{Q}^{g}(\mathbf{v}, \infty) \rightarrow P, \quad \operatorname{wt}(X)=-\sum_{i \in I} \mathbf{v}_{i} \alpha_{i} \text { for } X \in B_{Q}^{g}(\mathbf{v}, \infty)
$$

and we set

$$
\varphi_{i}(X)=\varepsilon_{i}(X)+\left\langle h_{i}, \operatorname{wt}(X)\right\rangle .
$$

Let $B_{Q}^{g}(\infty)=\bigsqcup_{\mathbf{v}} B_{Q}^{g}(\mathbf{v}, \infty)$.

Proposition $3.4([7])$. The maps defined above make $B_{Q}^{g}(\infty)$ a crystal and it is isomorphic to the crystal $B_{Q}(\infty)$ associated to the crystal base of $U_{q}^{-}(\mathfrak{g}(Q))$. 


\section{Crystal action on Nakajima's Quiver varieties}

In this section, we review the realization of the crystal graph of integrable highest weight representations of $\mathfrak{g}(Q)$ via quiver varieties. See [16] for details, including proofs.

Let $\mathbf{w}, \mathbf{v}, \mathbf{v}^{\prime}, \mathbf{v}^{\prime \prime} \in\left(\mathbb{Z}_{\geq 0}\right)^{I}$ be such that $\mathbf{v}=\mathbf{v}^{\prime}+\mathbf{v}^{\prime \prime}$. Consider the maps

$$
\Lambda\left(\mathbf{v}^{\prime \prime} ; \mathbf{0}\right) \times \Lambda\left(\mathbf{v}^{\prime} ; \mathbf{w}\right) \stackrel{p_{1}}{\leftarrow} \tilde{\mathbf{F}}\left(\mathbf{v}, \mathbf{w} ; \mathbf{v}^{\prime \prime}\right) \stackrel{p_{2}}{\rightarrow} \mathbf{F}\left(\mathbf{v}, \mathbf{w} ; \mathbf{v}^{\prime \prime}\right) \stackrel{p_{3}}{\rightarrow} \Lambda(\mathbf{v} ; \mathbf{w}),
$$

where the notation is as follows. A point of $\mathbf{F}\left(\mathbf{v}, \mathbf{w} ; \mathbf{v}^{\prime \prime}\right)$ is a point $(x, t) \in \Lambda(\mathbf{v} ; \mathbf{w})$ together with an $I$-graded, $x$-stable subspace $\mathbf{S}$ of $\mathbf{V}$ such that $\operatorname{dim} \mathbf{S}=\mathbf{v}^{\prime}=\mathbf{v}-\mathbf{v}^{\prime \prime}$. A point of $\tilde{\mathbf{F}}\left(\mathbf{v}, \mathbf{w} ; \mathbf{v}^{\prime \prime}\right)$ is a point $(x, t, \mathbf{S})$ of $\mathbf{F}\left(\mathbf{v}, \mathbf{w} ; \mathbf{v}^{\prime \prime}\right)$ together with a collection of isomorphisms $R_{i}^{\prime}: \mathbf{V}_{i}^{\prime} \cong \mathbf{S}_{i}$ and $R_{i}^{\prime \prime}: \mathbf{V}_{i}^{\prime \prime} \cong \mathbf{V}_{i} / \mathbf{S}_{i}$ for each $i \in I$. Then we define $p_{2}\left(x, t, \mathbf{S}, R^{\prime}, R^{\prime \prime}\right)=(x, t, \mathbf{S}), p_{3}(x, t, \mathbf{S})=(x, t)$ and $p_{1}\left(x, t, \mathbf{S}, R^{\prime}, R^{\prime \prime}\right)=\left(x^{\prime \prime}, x^{\prime}, t^{\prime}\right)$ where $x^{\prime \prime}, x^{\prime}, t^{\prime}$ are determined by

$$
\begin{aligned}
R_{\mathrm{in}(h)}^{\prime} x_{h}^{\prime} & =x_{h} R_{\mathrm{out}(h)}^{\prime}: \mathbf{V}_{\text {out }(h)}^{\prime} \rightarrow \mathbf{S}_{\mathrm{in}(h)}, \\
t_{i}^{\prime} & =t_{i} R_{i}^{\prime}: \mathbf{V}_{i}^{\prime} \rightarrow \mathbf{W}_{i} \\
R_{\mathrm{in}(h)}^{\prime \prime} x_{h}^{\prime \prime} & =x_{h} R_{\mathrm{out}(h)}^{\prime \prime}: \mathbf{V}_{\operatorname{out}(h)}^{\prime \prime} \rightarrow \mathbf{V}_{\operatorname{in}(h)} / \mathbf{S}_{\operatorname{in}(h)} .
\end{aligned}
$$

It follows that $x^{\prime}$ and $x^{\prime \prime}$ are nilpotent.

Lemma 4.1 ([12, Lemma 10.3]). One has

$$
\left(p_{3} \circ p_{2}\right)^{-1}\left(\Lambda(\mathbf{v} ; \mathbf{w})^{s t}\right) \subset p_{1}^{-1}\left(\Lambda\left(\mathbf{v}^{\prime \prime} ; \mathbf{0}\right) \times \Lambda\left(\mathbf{v}^{\prime} ; \mathbf{w}\right)^{s t}\right) .
$$

Thus, we can restrict (4.1) to $\Lambda^{\text {st }}$, forget the $\Lambda\left(\mathbf{v}^{\prime \prime} ; \mathbf{0}\right)$-factor and consider the quotient by $G_{\mathbf{v}}, G_{\mathbf{v}^{\prime}}$. This yields the diagram

$$
\mathcal{L}\left(\mathbf{v}^{\prime}, \mathbf{w}\right) \stackrel{\pi_{1}}{\leftarrow} \mathcal{F}\left(\mathbf{v}, \mathbf{w} ; \mathbf{v}-\mathbf{v}^{\prime}\right) \stackrel{\pi_{2}}{\rightarrow} \mathcal{L}(\mathbf{v}, \mathbf{w}),
$$

where

$$
\mathcal{F}\left(\mathbf{v}, \mathbf{w} ; \mathbf{v}-\mathbf{v}^{\prime}\right) \stackrel{\text { def }}{=}\left\{(x, t, \mathbf{S}) \in \mathbf{F}\left(\mathbf{v}, \mathbf{w} ; \mathbf{v}-\mathbf{v}^{\prime}\right) \mid(x, t) \in \Lambda(\mathbf{v} ; \mathbf{w})^{\text {st }}\right\} / G_{\mathbf{v}} .
$$

For $i \in I$ define $\varepsilon_{i}: \Lambda(\mathbf{v} ; \mathbf{w}) \rightarrow \mathbb{Z}_{\geq 0}$ by

$$
\varepsilon_{i}((x, t))=\operatorname{dim}_{\mathbb{C}} \text { Coker }\left(\bigoplus_{h: \operatorname{in}(h)=i} V_{\text {out }(h)} \stackrel{\left(x_{h}\right)}{\longrightarrow} V_{i}\right) .
$$

Then, for $c \in \mathbb{Z}_{\geq 0}$, let

$$
\mathcal{L}(\mathbf{v}, \mathbf{w})_{i, c}=\left\{[x, t] \in \mathcal{L}(\mathbf{v}, \mathbf{w}) \mid \varepsilon_{i}((x, t))=c\right\}
$$

where $[x, t]$ denotes the $G_{\mathbf{v}}$-orbit through the point $(x, t)$. We see that $\mathcal{L}(\mathbf{v}, \mathbf{w})_{i, c}$ is a locally closed subvariety of $\mathcal{L}(\mathbf{v}, \mathbf{w})$.

Assume $\mathcal{L}(\mathbf{v}, \mathbf{w})_{i, c} \neq \emptyset$ and let $\mathbf{v}^{\prime}=\mathbf{v}-c \mathbf{e}^{i}$ where $\mathbf{e}_{j}^{i}=\delta_{i j}$. Then

$$
\pi_{1}^{-1}\left(\mathcal{L}\left(\mathbf{v}^{\prime}, \mathbf{w}\right)_{i, 0}\right)=\pi_{2}^{-1}\left(\mathcal{L}(\mathbf{v}, \mathbf{w})_{i, c}\right) .
$$

Let

$$
\mathcal{F}\left(\mathbf{v}, \mathbf{w} ; c \mathbf{e}^{i}\right)_{i, 0}=\pi_{1}^{-1}\left(\mathcal{L}\left(\mathbf{v}^{\prime}, \mathbf{w}\right)_{i, 0}\right)=\pi_{2}^{-1}\left(\mathcal{L}(\mathbf{v}, \mathbf{w})_{i, c}\right) .
$$

We then have the following diagram.

$$
\mathcal{L}\left(\mathbf{v}^{\prime}, \mathbf{w}\right)_{i, 0} \stackrel{\pi_{1}}{\longleftarrow} \mathcal{F}\left(\mathbf{v}, \mathbf{w} ; c \mathbf{e}^{i}\right)_{i, 0} \stackrel{\pi_{2}}{\longrightarrow} \mathcal{L}(\mathbf{v}, \mathbf{w})_{i, c}
$$

The restriction of $\pi_{2}$ to $\mathcal{F}\left(\mathbf{v}, \mathbf{w} ; c \mathbf{e}^{i}\right)_{i, 0}$ is an isomorphism since the only possible choice for the subspace $\mathbf{S}$ of $\mathbf{V}$ is to have $\mathbf{S}_{j}=\mathbf{V}_{j}$ for $j \neq i$ and $\mathbf{S}_{i}$ equal to the 
sum of the images of the $x_{h}$ with $\operatorname{in}(h)=i . \mathcal{L}\left(\mathbf{v}^{\prime}, \mathbf{w}\right)_{i, 0}$ is an open subvariety of $\mathcal{L}\left(\mathbf{v}^{\prime}, \mathbf{w}\right)$.

Lemma $4.2([16]) . \quad$ (1) For any $i \in I$,

$$
\mathcal{L}(\mathbf{0}, \mathbf{w})_{i, c}=\left\{\begin{array}{ll}
p t & \text { if } c=0 \\
\emptyset & \text { if } c>0
\end{array} .\right.
$$

(2) Suppose $\mathcal{L}(\mathbf{v}, \mathbf{w})_{i, c} \neq \emptyset$ and $\mathbf{v}^{\prime}=\mathbf{v}-c \mathbf{e}^{i}$. Then the fiber of the restriction of $\pi_{1}$ to $\mathcal{F}\left(\mathbf{v}, \mathbf{w} ; \mathbf{e}^{i}\right)_{i, 0}$ is isomorphic to a Grassmanian variety.

Corollary 4.3. Suppose $\mathcal{L}(\mathbf{v}, \mathbf{w})_{i, c} \neq \emptyset$. Then there is a 1-1 correspondence between the set of irreducible components of $\mathcal{L}\left(\mathbf{v}-c \mathbf{e}^{i}, \mathbf{w}\right)_{i, 0}$ and the set of irreducible components of $\mathcal{L}(\mathbf{v}, \mathbf{w})_{i, c}$.

Let $B_{Q}^{g}(\mathbf{v}, \mathbf{w})$ denote the set of irreducible components of $\mathcal{L}(\mathbf{v}, \mathbf{w})$ and let $B_{Q}^{g}(\mathbf{w})=$ $\bigsqcup_{\mathbf{v}} B_{Q}^{g}(\mathbf{v}, \mathbf{w})$. For $X \in B_{Q}^{g}(\mathbf{v}, \mathbf{w})$, let $\varepsilon_{i}(X)=\varepsilon_{i}((x, t))$ for a generic point $[x, t] \in$ $X$. Then for $p \in \mathbb{Z}_{\geq 0}$ define

$$
B_{Q}^{g}(\mathbf{v}, \mathbf{w})_{i, p}=\left\{X \in B_{Q}^{g}(\mathbf{v}, \mathbf{w}) \mid \varepsilon_{i}(X)=p\right\} .
$$

Then by Corollary 4.3, $B_{Q}^{g}\left(\mathbf{v}-c \mathbf{e}^{i}, \mathbf{w}\right)_{i, 0} \cong B_{Q}^{g}(\mathbf{v}, \mathbf{w})_{i, c}$.

Suppose that $\bar{X} \in B_{Q}^{g}\left(\mathbf{v}-c \mathbf{e}^{i}, \mathbf{w}\right)_{i, 0}$ corresponds to $X \in B_{Q}^{g}(\mathbf{v}, \mathbf{w})_{i, c}$ by the above isomorphism. Then we define maps

$$
\begin{array}{ll}
\tilde{f}_{i}^{c}: B_{Q}^{g}\left(\mathbf{v}-c \mathbf{e}^{i}, \mathbf{w}\right)_{i, 0} \rightarrow B_{Q}^{g}(\mathbf{v}, \mathbf{w})_{i, c}, & \tilde{f}_{i}^{c}(\bar{X})=X, \\
\tilde{e}_{i}^{c}: B_{Q}^{g}(\mathbf{v}, \mathbf{w})_{i, c} \rightarrow B_{Q}^{g}\left(\mathbf{v}-c \mathbf{e}^{i}, \mathbf{w}\right)_{i, 0}, & \tilde{e}_{i}^{c}(X)=\bar{X} .
\end{array}
$$

We then define the maps

$$
\tilde{e}_{i}, \tilde{f}_{i}: B_{Q}^{g}(\mathbf{w}) \rightarrow B_{Q}^{g}(\mathbf{w}) \sqcup\{0\}
$$

by

$$
\begin{aligned}
& \tilde{e}_{i}: B_{Q}^{g}(\mathbf{v}, \mathbf{w})_{i, c} \stackrel{\tilde{e}_{i}^{c}}{\longrightarrow} B_{Q}^{g}\left(\mathbf{v}-c \mathbf{e}^{i}, \mathbf{w}\right)_{i, 0} \stackrel{\tilde{f}_{i}^{c-1}}{\longrightarrow} B_{Q}^{g}\left(\mathbf{v}-\mathbf{e}^{i}, \mathbf{w}\right)_{i, c-1}, \\
& \tilde{f}_{i}: B_{Q}^{g}(\mathbf{v}, \mathbf{w})_{i, c} \stackrel{\tilde{e}_{i}^{c}}{\longrightarrow} B_{Q}^{g}\left(\mathbf{v}-c \mathbf{e}^{i}, \mathbf{w}\right)_{i, 0} \stackrel{\tilde{f}_{i}^{c+1}}{\longrightarrow} B_{Q}^{g}\left(\mathbf{v}+\mathbf{e}^{i}, \mathbf{w}\right)_{i, c+1} .
\end{aligned}
$$

We set $\tilde{e}_{i}(X)=0$ for $X \in B_{Q}^{g}(\mathbf{v}, \mathbf{w})_{i, 0}$ and $\tilde{f}_{i}(X)=0$ for $X \in B_{Q}^{g}(\mathbf{v}, \mathbf{w})_{i, c}$ with $B_{Q}^{g}\left(\mathbf{v}+\mathbf{e}^{i}, \mathbf{w}\right)_{i, c+1}=\emptyset$. We also define

$$
\begin{gathered}
\text { wt }: B_{Q}^{g}(\mathbf{w}) \rightarrow P, \quad \operatorname{wt}(X)=\sum_{i \in I}\left(\mathbf{w}_{i} \omega_{i}-\mathbf{v}_{i} \alpha_{i}\right) \text { for } X \in B_{Q}^{g}(\mathbf{v}, \mathbf{w}), \\
\varphi_{i}(X)=\varepsilon_{i}(X)+\left\langle h_{i}, \operatorname{wt}(X)\right\rangle .
\end{gathered}
$$

Recall that we can consider $\mathbf{w}$ to be an dominant integral weight by $\mathbf{w}=$ $\sum_{i} \mathbf{w}_{i} \omega_{i}$.

Proposition $4.4([16])$. Under the maps defined above, $B_{Q}^{g}(\mathbf{w})$ is a crystal and is isomorphic to the crystal $B_{Q}(\mathbf{w})$ associated to the crystal base of $V_{Q}(\mathbf{w})$, the highest weight $U_{q}(\mathfrak{g})$-module with highest weight $\mathbf{w}$. 


\section{The CRYstal of THE UNIVERSAl ENVELOPING ALGEBRA AND ADMissible AUTOMORPHISMS}

As before, let $Q$ be a quiver without vertex loops. The corresponding symmetric generalized Cartan matrix is the matrix $A$ indexed by $I$ with entries

$$
a_{i j}=\left\{\begin{array}{ll}
2 & i=j \\
-\#\{\text { edges joining vertices } i \text { and } j\} & i \neq j
\end{array} .\right.
$$

Note that by the word edge we mean unoriented edge (i.e. elements of $E$ ). Again, we let $\mathfrak{g}(Q)$ denote the associated symmetric Kac-Moody algebra, with root system $\Delta(Q)($ see $[5])$.

An admissible automorphism a of a (double) quiver $Q$ is an automorphism of the underlying graph such that no edge connects two vertices in the same a-orbit. Following [11] we construct a symmetric matrix $M$ indexed by the vertex a-orbits I. We let the $(\mathbf{i}, \mathbf{j})$ entry be

$m_{\mathbf{i j}}=\left\{\begin{array}{ll}2 \#\{\text { vertices in ith orbit }\} & \mathbf{i}=\mathbf{j} \\ -\#\{\text { edges joining a vertex in ith orbit and a vertex in } \mathbf{j t h} \text { orbit }\} & \mathbf{i} \neq \mathbf{j}\end{array}\right.$.

Then let

$$
d_{\mathbf{i}}=m_{\mathrm{ii}} / 2=\#\{\text { vertices in ith orbit }\}
$$

and set $D=\operatorname{diag}\left(d_{\mathbf{i}}\right)$. Then $C=D^{-1} M$ is a symmetrizable generalized Cartan matrix. Let $\Gamma$ denote the corresponding valued graph. That is, $\Gamma$ has vertex set $\mathbf{I}$ and whenever $c_{\mathbf{i j}} \neq 0$, we draw an edge connecting $\mathbf{i}$ and $\mathbf{j}$ equipped with the ordered pair $\left(\left|c_{\mathbf{j i}}\right|,\left|c_{\mathbf{i j}}\right|\right)$. It is known [11, Prop 14.1.2] that any symmetrizable generalized Cartan matrix (and corresponding valued graph) can be obtained from a pair $(Q, \mathbf{a})$ in this way. Note that the fact that $\mathbf{a}$ is admissible ensures that $\Gamma$ has no vertex loops. Let $\mathfrak{g}(\Gamma)$ be the Kac-Moody algebra associated to $C$, with root system $\Delta(\Gamma)$.

Let $(-,-)_{Q}$ and $(-,-)_{\Gamma}$ be the symmetric bilinear forms determined by the matrices $A$ and $M$ respectively. The automorphism a acts naturally on the root lattice $\mathbb{Z} I$ for $Q$, and $(-,-)_{Q}$ is a-invariant. There is a canonical bijection

$$
f:(\mathbb{Z} I)^{\mathbf{a}} \rightarrow \mathbb{Z} \mathbf{I}, \quad f(\alpha)_{\mathbf{i}}=\alpha_{i} \text { for any } i \in \mathbf{i},
$$

from the fixed points in the root lattice for $Q$ to the root lattice for $\Gamma$. We will often suppress the bijection $f$ and consider the root lattice of $\Gamma$ to be the fixed points in the root lattice for $Q$. In particular, we have the simple roots for $\Gamma$ given by

$$
\alpha_{\mathbf{i}}=\sum_{i \in \mathbf{i}} \alpha_{i}
$$

We also define

$$
h_{\mathbf{i}}=\frac{1}{d_{\mathbf{i}}} \sum_{i \in \mathbf{i}} h_{i}
$$

Then the entries of $C$ are given by $c_{\mathbf{i j}}=\left\langle\alpha_{\mathbf{i}}, h_{\mathbf{j}}\right\rangle$.

Recall that $B_{Q}(\infty)$ and $B_{Q}^{g}(\infty)$ are the algebraic and geometric crystals of $U_{q}^{-}(\mathfrak{g}(Q))$ respectively. We know that $B_{Q}(\infty) \cong B_{Q}^{g}(\infty)$. One of the goals of this paper is to define a geometric crystal $B_{\Gamma}^{g}(\infty)$ of $U_{q}^{-}(\mathfrak{g}(\Gamma))$.

We use the same notation wt, $\varepsilon_{i}, \varphi_{i}, \tilde{e}_{i}$ and $\tilde{f}_{i}, i \in I$, for the maps of the crystals $B_{Q}(\infty)$ and $B_{Q}^{g}(\infty)$. 
Lemma 5.1. Suppose $i$ and $j$ are in the same vertex orbit $\mathbf{i}$. Then

$$
\tilde{e}_{i} \tilde{e}_{j}=\tilde{e}_{j} \tilde{e}_{i}, \quad \tilde{f}_{i} \tilde{f}_{j}=\tilde{f}_{j} \tilde{f}_{i} .
$$

Proof. We first briefly recall the definition of the Kashiwara operators $\tilde{e}_{i}$ and $\tilde{f}_{i}$ in the algebraic setting. For any $P \in U_{q}^{-}(\mathfrak{g}(Q))$, there exist unique $Q, R \in U_{q}^{-}(\mathfrak{g}(Q))$ such that

$$
\left[e_{i}, P\right]=\frac{q^{h_{i}} Q-q^{-h_{i}} R}{q-q^{-1}} .
$$

We can therefore define an endomorphism $e_{i}^{\prime}$ of $U_{q}^{-}(\mathfrak{g}(Q))$ given by $e_{i}^{\prime}(P)=R$. According to [6] we have

$$
U_{q}^{-}(\mathfrak{g}(Q))=\bigoplus_{n \geq 0} f_{i}^{(n)} \operatorname{ker} e_{i}^{\prime}
$$

Recall that $f_{i}^{(n)}=f_{i}^{n} /[n]_{i}$ ! where $[n]_{i}=\left(q^{n}-q^{-n}\right) /\left(q-q^{-1}\right)$ and $[n]_{i} !=\prod_{k=1}^{n}[k]_{i}$. Then the Kashiwara operators are induced by the operators on $U_{q}^{-}(\mathfrak{g}(Q))$ given by

$$
\begin{aligned}
& \tilde{f}_{i}\left(f_{i}^{(n)} u\right)=f_{i}^{(n+1)} u, \\
& \tilde{e}_{i}\left(f_{i}^{(n)} u\right)=f_{i}^{(n-1)} u,
\end{aligned}
$$

for $u \in \operatorname{ker} e_{i}^{\prime}$, where $f_{i}^{(n)}=0$ for $n<0$.

Fix $w \in U_{q}^{-}(\mathfrak{g}(Q))$ and let $w=f_{i}^{(n)} u$ for $u \in \operatorname{ker} e_{i}^{\prime}$. Let $u=f_{j}^{(m)} v$ for $v \in \operatorname{ker} e_{j}^{\prime}$. Since $i$ and $j$ lie in the same a-orbit and $\mathbf{a}$ is an admissible automorphism, there is no edge joining the vertices $i$ and $j$. Therefore $\left[f_{i}, f_{j}\right]=\left[e_{i}, f_{j}\right]=\left[e_{j}, f_{i}\right]=0$ and so we have

$$
w=f_{i}^{(n)} f_{j}^{(m)} v=f_{j}^{(m)} f_{i}^{(n)} v, \quad v \in \operatorname{ker} e_{i}^{\prime} \cap \operatorname{ker} e_{j}^{\prime} .
$$

Also, since $\left[e_{i}, f_{j}\right]=\left[e_{j}, f_{i}\right]=0$, we have $f_{i}^{(l)} v \in \operatorname{ker} e_{j}^{\prime}$ and $f_{j}^{(l)} v \in \operatorname{ker} e_{i}^{\prime}$ for all $l$. Therefore,

$$
\begin{aligned}
\tilde{e}_{i} \tilde{e}_{j} w & =\tilde{e}_{i} \tilde{e}_{j}\left(f_{j}^{(m)} f_{i}^{(n)} v\right) \\
& =\tilde{e}_{i}\left(f_{j}^{(m-1)} f_{i}^{(n)} v\right) \\
& =\tilde{e}_{i}\left(f_{i}^{(n)} f_{j}^{(m-1)} v\right) \\
& =f_{i}^{(n-1)} f_{j}^{(m-1)} v \\
& =f_{j}^{(m-1)} f_{i}^{(n-1)} v \\
& =\tilde{e}_{j}\left(f_{j}^{(m)} f_{i}^{(n-1)} v\right) \\
& =\tilde{e}_{j}\left(f_{i}^{(n-1)} f_{j}^{(m)} v\right) \\
& =\tilde{e}_{j} \tilde{e}_{i}\left(f_{i}^{(n)} f_{j}^{(m)} v\right) \\
& =\tilde{e}_{j} \tilde{e}_{i} w .
\end{aligned}
$$

The proof that $\tilde{f}_{i} \tilde{f}_{j}=\tilde{f}_{j} \tilde{f}_{i}$ is analogous.

By Lemma 5.1, we can unambiguously define the operators

$$
\tilde{e}_{\mathbf{i}}=\prod_{i \in \mathbf{i}} \tilde{e}_{i}, \quad \tilde{f}_{\mathbf{i}}=\prod_{i \in \mathbf{i}} \tilde{f}_{i}, \quad \mathbf{i} \in \mathbf{I} .
$$


For $b \in B_{Q}(\infty)$ and $\mathbf{i} \in \mathbf{I}$, we also define

$$
\begin{gathered}
\varepsilon_{\mathbf{i}}(b)=\max \left\{k \geq 0 \mid \tilde{e}_{\mathbf{i}}^{k} b \neq 0\right\}, \\
\varphi_{\mathbf{i}}(b)=\varepsilon_{\mathbf{i}}(b)+\left\langle h_{\mathbf{i}}, \operatorname{wt}(b)\right\rangle .
\end{gathered}
$$

Let $B_{\Gamma}(\infty)$ be the subset of $B_{Q}(\infty)$ generated by the the $\tilde{f}_{\mathbf{i}}, \mathbf{i} \in \mathbf{I}$ acting on the highest weight element $b_{\infty}$ of $B_{Q}(\infty)$. Note that if we restrict the map wt : $B_{Q}(\infty) \rightarrow P(Q)$, where $P(Q)$ is the weight lattice of $\mathfrak{g}(Q)$, to the subset $B_{\Gamma}(\infty)$, then the image lies in the subset of $P(Q)$ that is invariant under the natural action of $\mathbf{a}$. Thus we can view it as a map wt : $B_{\Gamma}(\infty) \rightarrow P(\Gamma)$ where $P(\Gamma)$ is the weight lattice of $\mathfrak{g}(\Gamma)$.

Proposition 5.2. The set $B_{\Gamma}(\infty)$, along with the maps wt, $\varepsilon_{\mathbf{i}}, \varphi_{\mathbf{i}}, \tilde{e}_{\mathbf{i}}$ and $\tilde{f}_{\mathbf{i}}$ is a $\mathfrak{g}(\Gamma)$-crystal.

Proof. We have to show that Axioms (1)-(7) of [3, Definition 4.5.1] defining a crystal are satisfied. Axioms (1)-(6) follow easily from the axioms for the crystal $B_{Q}(\infty)$ and Axiom (7) is vacuous since we never have $\varphi_{\mathbf{i}}(b)=-\infty$.

We want to show that the crystal $B_{\Gamma}(\infty)$ is actually isomorphic to the crystal of $U_{q}^{-}(\mathfrak{g}(\Gamma))$.

Definition 5.3. For $i \in I, B_{i}$ is the crystal defined as follows.

$$
\begin{gathered}
B_{i}=\left\{b_{i}(n) \mid n \in \mathbb{Z}\right\}, \\
\operatorname{wt}\left(b_{i}(n)\right)=n \alpha_{i}, \\
\varphi_{i}\left(b_{i}(n)\right)=n, \quad \varepsilon_{i}\left(b_{i}(n)\right)=-n, \\
\varphi_{j}\left(b_{i}(n)\right)=\varepsilon_{j}\left(b_{i}(n)\right)=-\infty \text { for } i \neq j .
\end{gathered}
$$

The action of $\tilde{e}_{j}$ and $\tilde{f}_{j}$ is given by

$$
\begin{gathered}
\tilde{e}_{i}\left(b_{i}(n)\right)=b_{i}(n+1), \\
\tilde{f}_{i}\left(b_{i}(n)\right)=b_{i}(n-1), \\
\tilde{e}_{j}\left(b_{i}(n)\right)=\tilde{f}_{j}\left(b_{i}(n)\right)=0, i \neq j .
\end{gathered}
$$

Let $b_{i}=b_{i}(0)$. We similarly define $B_{\mathbf{i}}$ for $\mathbf{i} \in \mathbf{I}$.

We now consider an equivalent definition of $B_{\mathbf{i}}$ for $\mathbf{i} \in \mathbf{I}$. Let $\mathbf{i}=\left\{i_{1}, \ldots, i_{k}\right\}$ and define

$$
B_{\mathbf{i}}^{\prime}=\left\{b_{\mathbf{i}}^{\prime}(n):=b_{i_{1}}(n) \otimes \cdots \otimes b_{i_{k}}(n) \mid n \in \mathbb{Z}\right\} \subset B_{i_{1}} \otimes \cdots \otimes B_{i_{k}} .
$$

By the definition of the tensor product of crystals (see [3]), we have that

$$
\begin{gathered}
\varphi_{i}\left(b_{\mathbf{i}}^{\prime}(n)\right)=n, \quad \varepsilon_{i}\left(b_{\mathbf{i}}^{\prime}(n)\right)=-n \text { for } i \in \mathbf{i} \\
\varphi_{j}\left(b_{\mathbf{i}}^{\prime}(n)\right)=\varepsilon_{j}\left(b_{\mathbf{i}}^{\prime}(n)\right)=-\infty \text { for } j \notin \mathbf{i}
\end{gathered}
$$

Thus we define $\varepsilon_{\mathbf{i}}=\varepsilon_{i}$ and $\varphi_{\mathbf{i}}=\varphi_{i}$ for any $i \in \mathbf{i}$. Note also that

$$
\operatorname{wt}\left(b_{\mathbf{i}}^{\prime}(n)\right)=\sum_{i \in \mathbf{i}} \operatorname{wt}\left(b_{i}(n)\right)=\sum_{i \in \mathbf{i}} n \alpha_{i}=n \alpha_{\mathbf{i}} .
$$


It is also straightforward to verify from the definition of the tensor product of crystals that

$$
\begin{gathered}
\tilde{e}_{\mathbf{i}}\left(b_{\mathbf{i}}^{\prime}(n)\right)=b_{\mathbf{i}}^{\prime}(n+1) \\
\tilde{f}_{\mathbf{i}}\left(b_{\mathbf{i}}^{\prime}(n)\right)=b_{\mathbf{i}}^{\prime}(n-1) \\
\tilde{e}_{\mathbf{j}}\left(b_{\mathbf{i}}^{\prime}(n)\right)=\tilde{f}_{\mathbf{j}}\left(b_{\mathbf{i}}^{\prime}(n)\right)=0, \quad \mathbf{i} \neq \mathbf{j} .
\end{gathered}
$$

Thus we see that $B_{\mathbf{i}}^{\prime} \cong B_{\mathbf{i}}$ and henceforth we identify the two. In particular, we identify $b_{\mathbf{i}}^{\prime}(n)$ and $b_{\mathbf{i}}(n)$.

The following characterization of the crystal of a universal enveloping algebra will be useful.

Proposition $5.4([7])$. Let $B$ be $a \mathfrak{g}(\Gamma)$-crystal and $b_{0}$ an element of $B$ with weight zero. Assume the following conditions.

(1) $\operatorname{wt}(B) \subset Q(\Gamma)^{-}$

(2) $b_{0}$ is the unique element of $B$ with weight zero.

(3) $\varepsilon_{\mathbf{i}}\left(b_{0}\right)=0$ for every $\mathbf{i}$.

(4) $\varepsilon_{\mathbf{i}}(b) \in \mathbb{Z}$ for any $b$ and $\mathbf{i}$.

(5) For every $\mathbf{i}$, there exists a strict embedding $\Psi_{\mathbf{i}}: B \rightarrow B \otimes B_{\mathbf{i}}$.

(6) $\Psi_{\mathbf{i}}(B) \subset B \times\left\{\tilde{f}_{\mathbf{i}}^{n} b_{\mathbf{i}} \mid n \geq 0\right\}$.

(7) For any $b \in B$ such that $b \neq b_{0}$ there exists $\mathbf{i}$ such that $\Psi_{\mathbf{i}}(b)=b^{\prime} \otimes \tilde{f}_{i}^{n} b_{\mathbf{i}}$ with $n>0$.

Then $B$ is isomorphic to the crystal associated to the crystal base of $U_{q}^{-}(\mathfrak{g}(\Gamma))$. The same result holds for $U_{q}^{-}(\mathfrak{g}(Q))$ if we replace $\mathbf{I}$ by $I$ (and $\mathbf{i}$ by $i$ ) in the above.

Proposition 5.5. $B_{\Gamma}(\infty)$ is isomorphic to the crystal associated to the crystal base of $U_{q}^{-}(\mathfrak{g}(\Gamma))$.

Proof. We prove this by an appeal to Proposition 5.4. Let $b_{0}=b_{\infty}$ be the unique element of $B_{\Gamma}(\infty)$ of weight zero. Conditions (1)-(4) are obvious. For $\mathbf{i}=\left\{i_{1}, \ldots, i_{d}\right\} \in \mathbf{I}$, we define

$$
\Psi_{\mathbf{i}}=\Psi_{i_{1}} \circ \Psi_{i_{2}} \circ \cdots \circ \Psi_{i_{d}}: B \rightarrow B \otimes B_{i_{1}} \otimes B_{i_{2}} \otimes \cdots \otimes B_{i_{d}} .
$$

Now, by conditions (1), (2) and (6) for the case of $U_{g}^{-}(\mathfrak{g}(Q))$, we see that $\Psi_{i}\left(b_{\infty}\right)=$ $b_{\infty} \otimes b_{i}$. Thus

$$
\Psi_{\mathbf{i}}\left(b_{0}\right)=\Psi_{\mathbf{i}}\left(b_{\infty}\right)=b_{\infty} \otimes b_{i_{1}} \otimes \cdots \otimes b_{i_{d}}=b_{\infty} \otimes b_{\mathbf{i}} \in B_{\Gamma}(\infty) \otimes B_{\mathbf{i}} .
$$

Thus, since each $\Psi_{i_{k}}$ is strict (that is, it commutes with all $\tilde{e}_{j}$ and $\tilde{f}_{j}$ ), we see that the image of $\Psi_{\mathbf{i}}$ restricted to $B_{\Gamma}(\infty)$ lies in $B_{\Gamma}(\infty) \otimes B_{\mathbf{i}}$. We also denote the restriction by $\Psi_{\mathbf{i}}$. Thus, condition (5) is satisfied.

Condition (6) for $\Psi_{\mathbf{i}}$ now follows from the corresponding conditions for the $\Psi_{i_{k}}$. For $b \in B_{\Gamma}(\infty)$ such that $b \neq b_{0}$, pick an $i$ such that condition (7) holds for $U_{q}^{-}(\mathfrak{g}(Q))$. Then it follows that condition $(7)$ holds for $U_{q}^{-}(\mathfrak{g}(\Gamma))$ for the orbit $\mathbf{i}$ containing $i$.

\section{A geometric Realization of the CRystal of the Universal}

ENVELOPING ALGEBRA IN THE NON-SIMPLY LACED CASE

Since we know that the geometric crystal $B_{Q}^{g}(\infty)$ is isomorphic to the algebraic crystal $B_{Q}(\infty)$, we can define $B_{\Gamma}^{g}(\infty)$ just as we defined $B_{\Gamma}(\infty)$ and we know that $B_{\Gamma}^{g}(\infty)$ is isomorphic to the crystal associated to the crystal base of $U_{q}^{-}(\mathfrak{g}(\Gamma))$. 
By definition, the underlying set of $B_{\Gamma}^{g}(\infty)$ consists of the irreducible components of Lusztig's quiver variety generated from the highest weight component (which consists of a point) by the action of the operators $\tilde{f}_{\mathbf{i}}$ for $\mathbf{i} \in \mathbf{I}$. We now seek to describe this set in a more direct and geometric way.

As described in [4], the automorphism a gives rise to an autoequivalence of the category of representations of the quiver as follows. Recall our quiver $Q$ with vertices $I$, edges $E$, and oriented edges $H$. Note that the automorphism a acts naturally on $H$. By a representation of the quiver $Q$, we mean a collection of vector spaces $\mathbf{V}=\left(\mathbf{V}_{i}\right)_{i \in I}$ and linear maps $\left(x_{h}: \mathbf{V}_{\text {out }(h)} \rightarrow \mathbf{V}_{\text {in }(h)}\right)_{h \in H}$. The dimension vector $\mathbf{v}=\operatorname{dim} \mathbf{V} \in \mathbb{Z} I$ is defined to be $\sum_{i \in I}\left(\operatorname{dim} \mathbf{V}_{i}\right) \alpha_{i}$ where $\left\{\alpha_{i}\right\}_{i \in I}$ is viewed as the standard basis of $\mathbb{Z} I$ or the set of simple roots depending on the context.

Given a representation of $Q$, we define a new representation as follows. The new collection of vector spaces is ${ }^{\mathrm{a}} \mathbf{V}$ where $\left({ }^{\mathbf{a}} \mathbf{V}\right)_{i}=\mathbf{V}_{\mathbf{a}^{-1}(i)}$ and the maps are given by $\left({ }^{\mathbf{a}} x\right)_{h}=x_{\mathbf{a}^{-1}(h)}$. We also have a natural action on morphisms between representations but we will not need this action. Thus we have a functor $F(\mathbf{a})$ on the category of representations. The functor $F(\mathbf{a})$ is an autoequivalence of this category. Note that in [4], the case of the single quiver (one orientation per edge) is considered while we are dealing with the double quiver here.

Note that $\operatorname{dim}^{\mathbf{a}} \mathbf{V}=\mathbf{a}(\operatorname{dim} \mathbf{V})$. So if we restrict ourselves to those $\mathbf{V}$ whose dimensions are invariant under $\mathbf{a}$, we can consider $F(\mathbf{a})$ as an automorphism of the space of representations. This induces an automorphism of Lusztig's quiver variety and thus an automorphism of the set of irreducible components (we assume that our chosen orientation $\Omega$ is compatible with the automorphism of the quiver which is always possible by $[11, \S 12.1 .1])$. We denote the automorphism of the set of irreducible components thus obtained by a.

Proposition 6.1. The underlying set of $B_{\Gamma}^{g}(\infty)$ is precisely the subset of irreducible components of $B_{Q}^{g}(\infty)$ consisting of those components that are invariant under the automorphism $\mathbf{a}$.

One should note that the irreducible components invariant under a are not necessarily invariant under $F(\mathbf{a})$. That is, each individual point in the irreducible component is not necessarily invariant under $F(\mathbf{a})$.

Proof. Let $B^{\prime}$ be the set of irreducible components invariant under a. It is easy to see that for $i \in I$, we have

$$
a \tilde{f}_{i} a^{-1}=\tilde{f}_{a(i)} .
$$

Thus for $\mathbf{i} \in \mathbf{I}$,

$$
\begin{aligned}
a \tilde{f}_{\mathbf{i}} a^{-1} & =a\left(\prod_{i \in \mathbf{i}} \tilde{f}_{i}\right) a^{-1}=\prod_{i \in \mathbf{i}}\left(a \tilde{f}_{i} a^{-1}\right) \\
& =\prod_{i \in \mathbf{i}} \tilde{f}_{a(i)}=\prod_{i \in \mathbf{i}} \tilde{f}_{i}=\tilde{f}_{\mathbf{i}} .
\end{aligned}
$$

Therefore, since the highest weight element of $B_{\Gamma}^{g}(\infty)$, which we will denote by $X_{\infty}$, is invariant under a, we have that $B_{\Gamma}^{g}(\infty) \subset B^{\prime}$ (as sets).

Now, let $X$ be an element of $B_{Q}^{g}(\infty)$ such that $\mathbf{a}(X)=X$. Let us order the set I. So we have $\mathbf{I}=\left\{\mathbf{i}_{1}, \ldots, \mathbf{i}_{l}\right\}$ and we let $\mathbf{i}_{p}=\left\{i_{p 1}, \ldots, i_{p d_{p}}\right\}$. Where $d_{p}=d_{\mathbf{i}_{p}}$ is the 
number of vertices in the orbit $\mathbf{i}_{p}$. We define a collection of non-negative integers $\mathbf{n}=\left(n_{j}^{b, c}\right)_{1 \leq j \leq k, 1 \leq b \leq l, 1 \leq c \leq d_{b}}$ inductively as follows.

$$
\begin{aligned}
n_{1}^{1,1} & =\varepsilon_{i_{11}}(X), \\
X_{j}^{b, c} & =\tilde{e}_{i_{b c}}^{n_{j}^{b, c}} \cdots \tilde{e}_{i_{b 1}}^{n_{j}^{b, 1}} \cdots \tilde{e}_{i_{1 d_{1}}}^{n_{j}^{1, d_{1}}} \cdots \tilde{e}_{i_{11}}^{n_{j}^{1,1}} \cdots \tilde{e}_{i_{l d_{l}}}^{n_{1}^{l, d_{l}}} \cdots \tilde{e}_{i_{l 1}}^{n_{1}^{l, 1}} \cdots \tilde{e}_{i_{1 d_{1}}}^{n_{1}^{1, d_{1}}} \cdots \tilde{e}_{i_{11}}^{n_{1}^{1,1}} X, \\
n_{j}^{b, c} & =\varepsilon_{i_{b c}}\left(X_{j}^{b, c-1}\right) \text { if } c>1, \\
n_{j}^{b, 1} & =\varepsilon_{i_{b 1}}\left(X_{j}^{b-1, d_{b-1}}\right) \text { if } b>1, \\
n_{j}^{1,1} & =\varepsilon_{i_{11}}\left(X_{j-1}^{l, d_{l}}\right) \text { if } j>1 .
\end{aligned}
$$

and $k$ is chosen such that $X_{k}^{l, d_{l}}=X_{\infty}$. Intuitively speaking, we are applying $\tilde{e}_{i}$ for $i \in \mathbf{i}_{1}$ as many times as possible (without getting 0 ). Then we apply $\tilde{e}_{i}$ for $i \in \mathbf{i}_{2}$ as many times as possible, etc. Once we have applied $\tilde{e}_{i}$ for $i \in \mathbf{i}_{l}$, we start again at $\mathbf{i}_{1}$. We continue this process until we obtain $X_{\infty}$, which we will always do since $B_{Q}^{g}(\infty)$ is connected. Then the $n_{j}^{b, c}$ tell us how many times we applied the various $\tilde{e}_{i}$. Note that it does not really matter what order we pick the various $i$ in each i since such $\tilde{e}_{i}$ commute. Also note that $X$ uniquely determines $\mathbf{n}$ and vice versa since using $\mathbf{n}$ we can apply the operators $\tilde{f}_{i}$ to $X_{\infty}$ to obtain $X$.

Identify $\mathbf{n}$ with the sequence

$$
\begin{aligned}
\mathbf{n}=\left(i_{l d_{l}} n_{k}^{l, d_{l}}\right. & \ldots, i_{l 1} n_{k}^{n^{l, 1}}, \ldots, i_{1 d_{1}} n_{k}^{1, d_{1}}, \ldots, i_{11} n_{k}^{1,1}, \ldots, \\
& \left.i_{l d_{l}}{ }^{l, d_{l}}, \ldots, i_{l 1}{ }^{n_{1}^{l, 1}}, \ldots, i_{1 d_{1}}^{n_{1}^{1, d_{1}}}, \ldots, i_{12}{ }^{n_{1}^{1,2}}, i_{11} n^{n_{1}^{1,1}}\right),
\end{aligned}
$$

where $i^{j}$ means the entry $i$ appears $j$ times. We define $\mathbf{n}_{j}^{b}$ to be the subsequence

$$
\mathbf{n}_{j}^{b}=\left(i_{b d_{b}}^{n_{j}^{b, d_{b}}}, \ldots, i_{b 1}^{n_{j}^{b, 1}}\right) .
$$

For a sequence $\left(i_{1}, \ldots, i_{m}\right)$, let

$$
\begin{aligned}
& \tilde{e}_{i_{1}, \ldots, i_{m}}=\tilde{e}_{i_{1}} \ldots \tilde{e}_{i_{m}} \\
& \tilde{f}_{i_{1}, \ldots, i_{m}}=\tilde{f}_{i_{m}} \ldots \tilde{f}_{i_{1}} .
\end{aligned}
$$

Note the reversal of the order of the indices in the second equation. By definition, we have $X_{\infty}=\tilde{e}_{\mathbf{n}} X$ and $X=\tilde{f}_{\mathbf{n}} X_{\infty}$. We define the action of $\mathbf{a}$ on a sequence of vertices by $\mathbf{a}\left(i_{1}, \ldots, i_{m}\right)=\left(\mathbf{a}\left(i_{1}\right), \ldots, \mathbf{a}\left(i_{m}\right)\right)$. Let $\left|\mathbf{n}_{j}^{b}\right|=\sum_{c=1}^{d_{b}} n_{j}^{b, c}$ be the length of the subsequence $\mathbf{n}_{j}^{b}$. Note that

$$
\left|\mathbf{a}\left(\mathbf{n}_{j}^{b}\right)\right|=\left|\mathbf{n}_{j}^{b}\right|,
$$

for each $j$ and $b$. Let $\mathbf{n}^{\prime}$ be the sequence obtained from $\mathbf{a}(\mathbf{n})$ by reordering the vertices appearing in each subsequence $\mathbf{a}\left(\mathbf{n}_{j}^{b}\right)$ such that the vertices appear in the order $i_{b d_{b}}, \ldots i_{b 1}$. Note that this merely involves rearranging vertices in the same a-orbit and thus we have

$$
\tilde{e}_{\mathbf{n}^{\prime}}=\tilde{e}_{\mathbf{a}(\mathbf{n})}, \quad \tilde{f}_{\mathbf{n}^{\prime}}=\tilde{f}_{\mathbf{a}(\mathbf{n})},
$$

and $\left|\left(\mathbf{n}^{\prime}\right)_{j}^{b}\right|=\left|\mathbf{a}\left(\mathbf{n}_{j}^{b}\right)\right|=\left|\mathbf{n}_{j}^{b}\right|$. In particular, we have $\tilde{e}_{\mathbf{n}^{\prime}} X=X_{\infty}$. Thus, we must have $\left(n^{\prime}\right)_{1}^{1, c} \leq n_{1}^{1, c}$ for $1 \leq c \leq d_{1}$ by our definition of $n_{1}^{1, c}$ as the maximum number of times $\tilde{e}_{i_{1 c}}$ can be applied to $X$ without getting zero. It follows that $\left(n^{\prime}\right)_{1}^{1, c}=n_{1}^{1, c}$ for $1 \leq c \leq d_{1}$. We can then continue in this way to show that in fact $\mathbf{n}^{\prime}=\mathbf{n}$. Therefore, each subsequence $\mathbf{n}_{j}^{b}$ is invariant under the action of a after rearranging 
the terms. Thus we must have $n_{j}^{b, c}=n_{j}^{b, c^{\prime}}$ for $1 \leq c, c^{\prime} \leq d_{b}$. Denote this common value by $n_{j}^{b}$. It follows that

$$
\tilde{f}_{\mathbf{n}}=\tilde{f}_{\mathbf{i}_{l}}^{n_{k}^{l}} \ldots \tilde{f}_{\mathbf{i}_{1}}^{n_{k}^{1}} \ldots \tilde{f}_{\mathbf{i}_{l}}^{n_{1}^{l}} \ldots \tilde{f}_{\mathbf{i}_{1}}^{n_{1}^{1}}
$$

Therefore $X=\tilde{f}_{\mathbf{n}} X_{\infty} \in B_{\Gamma}^{g}(\infty)$. So $B^{\prime} \subset B_{\Gamma}^{g}(\infty)$ (as sets) which completes the proof.

Remark 6.2. For the case where $Q$ is of finite type, we have by Proposition 1.3 that the irreducible components $B_{Q}^{g}(\infty)$ are the closures of the conormal bundles of the various $G_{\mathbf{v}}$ orbits in $\mathbf{E}_{\Omega}(\mathbf{v})$. Then by a result of [4] one can see that the irreducible components fixed by a are precisely the closures of the conormal bundles to the orbits through the isomorphically invariant representations (that is, the representations which are invariant under $F(\mathbf{a})$ up to isomorphism).

We have realized the crystal of $U_{q}^{-}(\mathfrak{g}(\Gamma))$ geometrically on the set of irreducible components of Lusztig's quiver varieties associated to $Q$ which are invariant under the automorphism a. The crystal maps are defined in terms of those coming from the crystal of $U_{q}^{-}(\mathfrak{g}(Q))$. However, it is also possible to give a more direct geometric definition of these maps. We briefly sketch the construction.

Recall that $\alpha_{\mathbf{i}}=\sum_{i \in \mathbf{i}} \alpha_{i}$ and that no two vertices in the orbit $\mathbf{i}$ are connected by an edge. Define

$$
\mathbf{E}^{0}(\mathbf{v})_{\mathbf{i}, p}=\left\{x \in \mathbf{E}^{0}(\mathbf{v}) \mid \varepsilon_{i}(x)=p \forall i \in \mathbf{i}\right\} .
$$

Note that $\mathbf{E}^{0}(\mathbf{v})_{\mathbf{i}, p}$ is a locally closed subvariety of $\mathbf{E}^{0}(\mathbf{v})$. We have for a-stable $\mathbf{v}$

$$
F(\mathbf{a})\left(\mathbf{E}^{0}(\mathbf{v})_{i, p}\right)=\mathbf{E}^{0}(\mathbf{v})_{\mathbf{a}(i), p},
$$

and therefore

$$
\mathbf{E}^{0}(\mathbf{v})_{\mathbf{i}, p}=\bigcap_{i \in \mathbf{i}} \mathbf{E}^{0}(\mathbf{v})_{i, p}=\bigcap_{n=1}^{d_{\mathbf{i}}} F(\mathbf{a})^{n}\left(\mathbf{E}^{0}(\mathbf{v})_{i, p}\right) \text { for } i \in \mathbf{i} .
$$

Let $X \in B_{\Gamma}^{g}(\infty)$. Thus we have $\mathbf{a}(X)=X$. Then we see from $(6.1)$ that $\varepsilon_{i}(X)=$ $\varepsilon_{j}(X)$ for $i, j \in \mathbf{i}$ and we define their common value by $\varepsilon_{\mathbf{i}}(X)$. It is easy to see that this definition coincides with the previous definition of $\varepsilon_{\mathbf{i}}(X)$.

Now consider the diagram (3.1) in the case $\mathbf{v}=\overline{\mathbf{v}}+c \alpha_{\mathbf{i}}$ for $c \in \mathbb{Z}_{\geq 0}$. One easily sees that $\mathbf{E}^{0}\left(c \alpha_{\mathbf{i}}\right)=\{0\}$. Thus we have

$$
\mathbf{E}^{0}(\overline{\mathbf{v}}) \cong \mathbf{E}^{0}(\overline{\mathbf{v}}) \times \mathbf{E}^{0}\left(c \alpha_{\mathbf{i}}\right) \stackrel{\varpi_{1}}{\longleftarrow} \mathbf{E}^{0}\left(\overline{\mathbf{v}}, c \alpha_{\mathbf{i}}\right) \stackrel{\varpi_{2}}{\longrightarrow} \mathbf{E}^{0}(\mathbf{v}) .
$$

Using this diagram, we proceed exactly as in the case of the quiver $Q$ and see that $B_{\Gamma}^{g}(\overline{\mathbf{v}}, \infty)_{\mathbf{i}, 0} \cong B_{\Gamma}^{g}(\mathbf{v}, \infty)_{\mathbf{i}, c}$ where $B_{\Gamma}^{g}(\mathbf{v}, \infty)_{\mathbf{i}, p}$ is the set of all $X \in B_{\Gamma}^{g}(\mathbf{v}, \infty)$ such that $\varepsilon_{\mathbf{i}}(X)=p$. Then we can define $\tilde{e}_{\mathbf{i}}$ and $\tilde{f}_{\mathbf{i}}$ just as we defined $\tilde{e}_{i}$ and $\tilde{f}_{i}$ and it is not hard to see that these direct geometric definitions agree with the above definitions in terms of compositions of the $\tilde{e}_{i}$ and $\tilde{f}_{i}$.

Let $\Lambda^{\mathbf{a}}(\mathbf{v})$ be the subvariety of $\Lambda(\mathbf{v})$ consisting of the union of those irreducible components of $\Lambda(\mathbf{v})$ that are invariant under $\mathbf{a}$. The above results can then be collected into the following.

Theorem 6.3. Let $B_{\Gamma}^{g}(\mathbf{v}, \infty)$ be the set of irreducible components of $\Lambda^{\mathbf{a}}(\mathbf{v})$ and let $B_{\Gamma}^{g}(\infty)=\bigsqcup_{\mathbf{v}} B_{\Gamma}^{g}(\mathbf{v}, \infty)$. The maps $\tilde{e}_{\mathbf{i}}, \tilde{f}_{\mathbf{i}}$, wt, $\varepsilon_{\mathbf{i}}$ and $\varphi_{\mathbf{i}}, \mathbf{i} \in \mathbf{I}$, defined above endow $B_{\Gamma}^{g}(\infty)$ with the structure of a crystal and this crystal is isomorphic to that associated to the crystal base of $U_{q}^{-}(\mathfrak{g}(\Gamma))$. 


\section{The CRYSTAL OF AN IRREDUCIBLE HigheST-WEIGHT REPRESENTATION AND ADMISSIBLE AUTOMORPHISMS}

In this section and the next we apply our methods to the irreducible highest weight representations.

Let $\mathbf{w} \in P(Q)^{+}$be a dominant integral weight of $\mathfrak{g}(Q)$ such that $\mathbf{a}(\mathbf{w})=\mathbf{w}$. Thus we can also think of $\mathbf{w}$ as a dominant integral weight of $\mathfrak{g}(\Gamma)$. Recall that $B_{Q}(\mathbf{w})$ and $B_{Q}^{g}(\mathbf{w})$ are the algebraic and geometric crystals of $V_{Q}(\mathbf{w})$, the irreducible highest weight representation of $U_{q}(\mathfrak{g}(Q))$ of highest weight $\mathbf{w}$, respectively. We know that $B_{Q}(\mathbf{w}) \cong B_{Q}^{g}(\mathbf{w})$. Let $B_{\Gamma}(\mathbf{w})^{\prime}$ denote the crystal of $V_{\Gamma}(\mathbf{w})$, the irreducible highest weight module of $U_{q}(\mathfrak{g}(\Gamma))$ of highest weight $\mathbf{w}$. Our goal is to define a geometric crystal $B_{\Gamma}^{g}(\mathbf{w})$ of $V_{\Gamma}(\mathbf{w})$.

As for the case of the universal enveloping algebra, the operators $\tilde{e}_{i}$ and $\tilde{e}_{j}$ (resp. $\tilde{f}_{i}$ and $\tilde{f}_{j}$ ) for $i, j \in \mathbf{i}$ commute and we define the operators

$$
\tilde{e}_{\mathbf{i}}=\prod_{i \in \mathbf{i}} \tilde{e}_{i}, \quad \tilde{f}_{\mathbf{i}}=\prod_{i \in \mathbf{i}} \tilde{f}_{i}, \quad \mathbf{i} \in \mathbf{I} .
$$

For $b \in B_{Q}(\mathbf{w})$ and $\mathbf{i} \in \mathbf{I}$, we also define as before

$$
\begin{gathered}
\varepsilon_{\mathbf{i}}(b)=\max \left\{k \geq 0 \mid \tilde{e}_{\mathbf{i}}^{k} b \neq 0\right\}, \\
\varphi_{\mathbf{i}}(b)=\varepsilon_{\mathbf{i}}(b)+\left\langle h_{\mathbf{i}}, \operatorname{wt}(b)\right\rangle .
\end{gathered}
$$

Let $B_{\Gamma}(\mathbf{w})$ be the subset of $B_{Q}(\mathbf{w})$ generated by the the $\tilde{f}_{\mathbf{i}}, \mathbf{i} \in \mathbf{I}$ acting on the highest weight element $b_{\mathbf{w}}$ of $B_{Q}(\mathbf{w})$. Note that if we restrict the map wt : $B_{Q}(\mathbf{w}) \rightarrow P(Q)$, where $P(Q)$ is the weight lattice of $\mathfrak{g}(Q)$, to the subset $B_{\Gamma}(\mathbf{w})$, then the image lies in the subset of $P(Q)$ that is invariant under the action of $\mathbf{a}$. Thus we can view it as a map wt $: B_{\Gamma}(\mathbf{w}) \rightarrow P(\Gamma)$ where $P(\Gamma)$ is the weight lattice of $\mathfrak{g}(\Gamma)$.

Note that for $b \in B_{\Gamma}(\mathbf{w}), \operatorname{wt}(b)$ is invariant under $\mathbf{a}$ and $\varepsilon_{\mathbf{i}}(b)=\varepsilon_{i}(b)$ for any $i \in \mathbf{i}$. Thus

$$
\begin{aligned}
\varphi_{\mathbf{i}}(b) & =\varepsilon_{\mathbf{i}}(b)+\left\langle h_{\mathbf{i}}, \operatorname{wt}(b)\right\rangle \\
& =\varepsilon_{\mathbf{i}}(b)+\frac{1}{d_{\mathbf{i}}} \sum_{i \in \mathbf{i}}\left\langle h_{i}, \operatorname{wt}(b)\right\rangle \\
& =\varepsilon_{i}(b)+\left\langle h_{i}, \operatorname{wt}(b)\right\rangle \text { for some } i \in \mathbf{i} \\
& =\max \left\{k \geq 0 \mid \tilde{f}_{i}^{k} b \neq 0\right\} \\
& =\max \left\{k \geq 0 \mid \tilde{f}_{\mathbf{i}}^{k} b \neq 0\right\} .
\end{aligned}
$$

Proposition 7.1. The set $B_{\Gamma}(\mathbf{w})$, along with the maps wt, $\varepsilon_{\mathbf{i}}, \varphi_{\mathbf{i}}, \tilde{e}_{\mathbf{i}}$ and $\tilde{f}_{\mathbf{i}}$ is a $\mathfrak{g}(\Gamma)$-crystal.

Proof. We have to show that Axioms (1)-(7) of [3, Definition 4.5.1] defining a crystal are satisfied. Axioms (1)-(6) follow easily from the axioms for the crystal $B_{Q}(\mathbf{w})$ and Axiom (7) is vacuous since we never have $\varphi_{\mathbf{i}}(b)=-\infty$.

We want to show that the crystal $B_{\Gamma}(\mathbf{w})$ is actually isomorphic to the crystal $B_{\Gamma}(\mathbf{w})^{\prime}$.

Definition 7.2. For $\mathbf{w} \in P(Q)^{+}$(resp. $\left.\mathbf{w} \in P(\Gamma)^{+}\right), T_{\mathbf{w}}=\left\{t_{\mathbf{w}}\right\}$ is the crystal with one element where $\operatorname{wt}\left(t_{\mathbf{w}}\right)=\mathbf{w}, \varepsilon_{i}\left(t_{\mathbf{w}}\right)=\varphi_{i}\left(t_{\mathbf{w}}\right)=-\infty\left(\right.$ resp. $\varepsilon_{\mathbf{i}}\left(t_{\mathbf{w}}\right)=\varphi_{\mathbf{i}}\left(t_{\mathbf{w}}\right)=$ $-\infty)$ and $\tilde{e}_{i}\left(t_{\mathbf{w}}\right)=\tilde{f}_{i}\left(t_{\mathbf{w}}\right)=0$ for all $i \in I$ (resp. $\tilde{e}_{\mathbf{i}}\left(t_{\mathbf{w}}\right)=\tilde{f}_{\mathbf{i}}\left(t_{\mathbf{w}}\right)=0$ for all $\left.\mathbf{i} \in \mathbf{I}\right)$. 
Note that if we view $\mathbf{w} \in P(\Gamma)^{+}$as an a-invariant element of $P(Q)^{+}$, we have that the definitions of $T_{\mathbf{w}}$ for $\mathfrak{g}(Q)$ and $\mathfrak{g}(\Gamma)$ coincide under the above definitions of $\tilde{e}_{\mathbf{i}}$ and $\tilde{f}_{\mathbf{i}}$ as the composition of various $\tilde{e}_{i}$ and $\tilde{f}_{i}$.

Let $B(\mathbf{w})$ denote either $B_{Q}(\mathbf{w})$ or $B_{\Gamma}(\mathbf{w})^{\prime}$ and $B(\infty)$ denote either $B_{Q}(\infty)$ or $B_{\Gamma}(\infty)$. We will use the notation $\iota$ to denote elements of either $I$ or $\mathbf{I}$. Now consider the tensor product of crystals $B(\infty) \otimes T_{\mathbf{w}}$ and let $\pi_{\mathbf{w}}: B(\infty) \otimes T_{\mathbf{w}} \rightarrow B(\mathbf{w})$ be the strict morphism given by $\tilde{f}_{\iota_{1}} \ldots \tilde{f}_{\iota_{l}} b_{\infty} \otimes t_{\mathbf{w}} \rightarrow \tilde{f}_{\iota_{1}} \ldots \tilde{f}_{\iota_{l}} b_{\mathbf{w}}$, where $b_{\infty}$ is the unique element of $B(\infty)$ of weight zero and $b_{\mathbf{w}}$ is the unique element of $B(\mathbf{w})$ of weight $\mathbf{w}$. We have that

$$
\pi_{\mathbf{w}}:\left\{b \in B(\infty) \otimes T_{\mathbf{w}} \mid \pi_{\mathbf{w}}(b) \neq 0\right\} \cong B(\mathbf{w}) .
$$

The following characterization of the crystal of an irreducible highest weight representation with be useful.

Proposition $7.3([7])$. Let $B$ be a $\mathfrak{g}(Q)$ or $\mathfrak{g}(\Gamma)$-crystal and $b_{\mathbf{w}}$ an element of $B$ with weight $\mathbf{w} \in P^{+}$. Assume the following conditions.

(1) $b_{\mathbf{w}}$ is the unique element of $B$ with weight $\mathbf{w}$.

(2) There is a strict morphism $\Phi: B(\infty) \otimes T_{\mathbf{w}} \rightarrow B$ such that $\Phi\left(b_{\infty} \otimes t_{\mathbf{w}}\right)=b_{\mathbf{w}}$.

(3) The set $\left\{b \in B(\infty) \otimes T_{\mathbf{w}} \mid \Phi(b) \neq 0\right\}$ is isomorphic to $B$ through $\Phi$ as a set.

(4) For any $b \in B, \varepsilon_{\iota}(b)=\max \left\{k \geq 0 \mid \tilde{e}_{\iota}^{k}(b) \neq 0\right\}$ and $\varphi_{\iota}(b)=\max \{k \geq$ $\left.0 \mid \tilde{f}_{\iota}^{k}(b) \neq 0\right\}$ for all $\iota$.

Then $B$ is isomorphic to $B(\mathbf{w})$.

Proposition 7.4. We have $B_{\Gamma}(\mathbf{w}) \cong B_{\Gamma}(\mathbf{w})^{\prime}$.

Proof. We prove this by an appeal to Proposition 7.3, whose conditions are easily verified.

\section{A geOMEtric REALization OF the CRYSTAl OF AN IRREDUCible HIGHEST-WEIGHT REPRESENTATION IN THE NON-SIMPLY LACED CASE}

Since we know that the geometric crystal $B_{Q}^{g}(\mathbf{w})$ is isomorphic to the algebraic crystal $B_{Q}(\mathbf{w})$, we can define $B_{\Gamma}^{g}(\mathbf{w})$ just as we defined $B_{\Gamma}(\mathbf{w})$ and we know that $B_{\Gamma}^{g}(\mathbf{w})$ is isomorphic to the crystal associated to the crystal base of $V_{\Gamma}(\mathbf{w})$. By definition, the underlying set of $B_{\Gamma}^{g}(\mathbf{w})$ consists of the irreducible components of Lusztig's quiver varieties generated from the highest weight component (which consists of a point) by the action of the operators $\tilde{f}_{\mathbf{i}}$ for $\mathbf{i} \in I$. We now seek to describe this set in a more direct and geometric way.

We first extend the action of the automorphism a to Nakajima's quiver varieties as follows. Let $\mathbf{V}$ and $\mathbf{W}$ be $I$-graded vector spaces of graded dimension $\mathbf{v}$ and $\mathbf{w}$ respectively. As before, we define the new vector spaces ${ }^{\mathbf{a}} \mathbf{V}$ and ${ }^{\mathbf{a}} \mathbf{W}$ and we have the action $F(\mathbf{a}): \Lambda(\mathbf{v}) \rightarrow \Lambda(\mathbf{a}(\mathbf{v}))$ on Lusztig's quiver varieties. For an element $t=\left(t_{i}\right) \in \bigoplus_{i \in I} \operatorname{Hom}\left(\mathbf{V}_{i}, \mathbf{W}_{i}\right)$, we define a new element ${ }^{\mathbf{a}} t=\left({ }^{\mathbf{a}} t_{i}\right) \in$ $\bigoplus_{i \in I} \operatorname{Hom}\left({ }^{\mathbf{a}} \mathbf{V}_{i},{ }^{\mathbf{a}} \mathbf{W}_{i}\right)$ by ${ }^{\mathbf{a}} t_{i}=t_{\mathbf{a}^{-1}(i)}$. Combining these action yields a map

$$
F(\mathbf{a}): \Lambda(\mathbf{v}, \mathbf{w}) \rightarrow \Lambda(\mathbf{a}(\mathbf{v}), \mathbf{a}(\mathbf{w})),
$$

which in turn induces a map

$$
F(\mathbf{a}): \mathcal{L}(\mathbf{v}, \mathbf{w}) \rightarrow \mathcal{L}(\mathbf{a}(\mathbf{v}), \mathbf{a}(\mathbf{w})) .
$$

Note that if we restrict ourselves to $V$ and $W$ whose dimensions are invariant under a, we can consider $F(\mathbf{a})$ as an automorphism of Nakajima's quiver variety and thus 
an automorphism of the set of irreducible components. As before, we denote the automorphism of the set of irreducible components thus obtained by $\mathbf{a}$.

Proposition 8.1. The underlying set of $B_{\Gamma}^{g}(\mathbf{w})$ is precisely the subset of irreducible components of $B_{Q}^{g}(\mathbf{w})$ consisting of those components that are invariant under the automorphism $\mathbf{a}$.

Proof. The proof is almost exactly analogous to that of Proposition 6.1 and will be omitted.

As in the case of Lusztig's quiver variety, we should note that the irreducible components invariant under a are not necessarily invariant under $F(\mathbf{a})$. That is, each individual point in the irreducible component is not necessarily invariant under $F(\mathbf{a})$.

We have realized the crystal of $V_{\Gamma}(\mathbf{w})$ geometrically on the set of irreducible components of the Lusztig quiver variety associated to $Q$ which are invariant under the automorphism a. The crystal maps are defined in terms of those coming from the crystal of $V_{Q}(\mathbf{w})$. However, as for the case of the universal enveloping algebra, it is also possible to give a more direct geometric definition of these maps. We briefly sketch the construction.

Recall that $\alpha_{\mathbf{i}}=\sum_{i \in \mathbf{i}} \alpha_{i}$ and that no two vertices in the orbit $\mathbf{i}$ are connected by an edge. Define

$$
\mathcal{L}(\mathbf{v}, \mathbf{w})_{\mathbf{i}, p}=\left\{[x, t] \in L(\mathbf{v}, \mathbf{w}) \mid \varepsilon_{i}((x, t))=p \forall i \in \mathbf{i}\right\} .
$$

Note that $\mathcal{L}(\mathbf{v}, \mathbf{w})_{\mathbf{i}, p}$ is a locally closed subvariety of $\mathcal{L}(\mathbf{v}, \mathbf{w})$. We have for a-stable $\mathbf{v}$ and $\mathbf{w}$

$$
F(\mathbf{a})\left(\mathcal{L}(\mathbf{v}, \mathbf{w})_{\mathbf{i}, p}\right)=\mathcal{L}(\mathbf{v}, \mathbf{w})_{\mathbf{a}(i), p}
$$

and therefore

$$
\mathcal{L}(\mathbf{v}, \mathbf{w})_{\mathbf{i}, p}=\bigcap_{i \in \mathbf{i}} \mathcal{L}(\mathbf{v}, \mathbf{w})_{i, p}=\bigcap_{n=1}^{d_{\mathbf{i}}} F(\mathbf{a})^{n}\left(\mathcal{L}(\mathbf{v}, \mathbf{w})_{i, p}\right) \text { for } i \in \mathbf{i} .
$$

Let $X \in B_{\Gamma}^{g}(\mathbf{w})$. Thus we have $\mathbf{a}(X)=X$. Then we see from (8.1) that $\varepsilon_{i}(X)=$ $\varepsilon_{j}(X)$ for $i, j \in \mathbf{i}$ and we define their common value by $\varepsilon_{\mathbf{i}}(X)$. It is easy to see that this definition coincides with the previous definition of $\varepsilon_{\mathbf{i}}(X)$.

Now consider the diagram (4.2) in the case $\mathbf{v}=\mathbf{v}^{\prime}+c \alpha_{\mathbf{i}}$ for $c \in \mathbb{Z}_{\geq 0}$. Then we have

$$
\mathcal{L}\left(\mathbf{v}^{\prime}, \mathbf{w}\right) \stackrel{\pi_{1}}{\longleftarrow} \mathcal{F}\left(\mathbf{v}, \mathbf{w}, c \alpha_{\mathbf{i}}\right) \stackrel{\pi_{2}}{\longrightarrow} \mathcal{L}(\mathbf{v}, \mathbf{w})
$$

Using this diagram, we proceed exactly as in the case of the quiver $Q$ and see that $B_{\Gamma}^{g}\left(\mathbf{v}^{\prime}, \mathbf{w}\right)_{\mathbf{i}, 0} \cong B_{\Gamma}^{g}(\mathbf{v}, \mathbf{w})_{\mathbf{i}, c}$ where $B_{\Gamma}^{g}(\mathbf{v}, \mathbf{w})_{\mathbf{i}, p}$ is the set of all $X \in B_{\Gamma}^{g}(\mathbf{v}, \mathbf{w})$ such that $\varepsilon_{\mathbf{i}}(X)=p$. Then we can define $\tilde{e}_{\mathbf{i}}$ and $\tilde{f}_{\mathbf{i}}$ just as we defined $\tilde{e}_{i}$ and $\tilde{f}_{i}$ and it is not hard to see that these direct geometric definitions agree with the above definitions in terms of compositions of the $\tilde{e}_{i}$ and $\tilde{f}_{i}$.

Let $\mathcal{L}^{\mathbf{a}}(\mathbf{v}, \mathbf{w})$ be the subvariety of $\mathcal{L}(\mathbf{v}, \mathbf{w})$ consisting of the union of those irreducible components of $\mathcal{L}(\mathbf{v}, \mathbf{w})$ that are invariant under $\mathbf{a}$. The above results can then be collected into the following.

Theorem 8.2. Let $B_{\Gamma}^{g}(\mathbf{v}, \mathbf{w})$ be the set of irreducible components of $\mathcal{L}^{\mathbf{a}}(\mathbf{v}, \mathbf{w})$ and let $B_{\Gamma}^{g}(\mathbf{w})=\bigsqcup_{\mathbf{v}} B_{\Gamma}^{g}(\mathbf{v}, \mathbf{w})$. The maps $\tilde{e}_{\mathbf{i}}, \tilde{f}_{\mathbf{i}}, \mathbf{w t}, \varepsilon_{\mathbf{i}}$ and $\varphi_{\mathbf{i}}, \mathbf{i} \in \mathbf{I}$, defined above endow $B_{\Gamma}^{g}(\mathbf{w})$ with the structure of a crystal and this crystal is isomorphic to that 


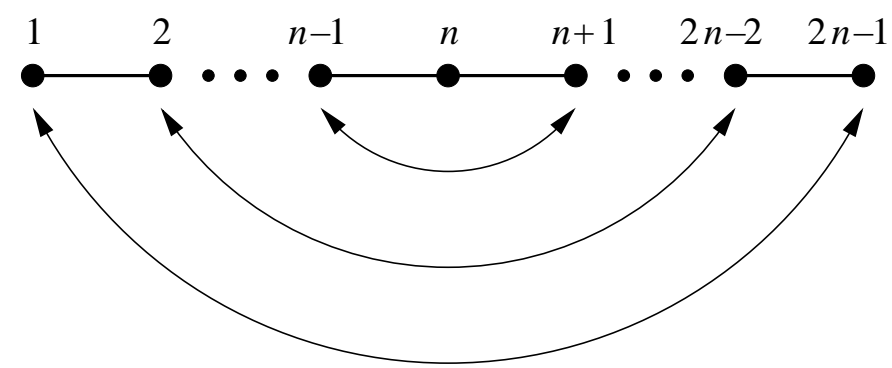

Figure 1. The admissible automorphism of the Dynkin diagram of type $A_{2 n-1}$ realizing the valued graph of type $B_{n}$. The arrows indicate the action of the automorphism.

associated to the crystal base of $V_{\Gamma}(\mathbf{w})$, the irreducible highest weight module of $U_{q}(\mathfrak{g}(\Gamma))$ of highest weight $\mathbf{w}$.

\section{A geometric Realization of the SPin Representation of $\mathfrak{s o}_{2 n+1}$}

In this section, we will apply the results of this paper to give an explicit realization of the spin representation of the Lie algebra $\mathfrak{s o}_{2 n+1}=\mathfrak{s o}_{2 n+1} \mathbb{C}$ of type $B_{n}$. In the process, we will end up with a combinatorial description of the crystal in terms of Young diagrams.

Let $\mathfrak{g}=\mathfrak{s o}_{2 n+1}$. The spin representation is the highest weight representation $V\left(\omega_{n}\right)$ of highest weight $\omega_{n}$. The valued graph, or Dynkin diagram, of type $B_{n}$ can be obtained from the Dynkin diagram of type $A_{2 n-1}$ and the automorphism a as depicted in Figure 1. We let $Q$ be the quiver of type $A_{2 n-1}$ (we pick an orientation compatible with the automorphism) and $\Gamma$ the corresponding valued graph of type $B_{n}$. Since we are interested in the spin representation, we should consider $\mathbf{w}=\mathbf{e}^{n}$ where $\mathbf{e}^{n} \in\left(\mathbb{Z}_{\geq 0}\right)^{2 n-1}$ is the element with $n$th component equal to one and all other components equal to zero. This element is invariant under the automorphism a.

Let $\mathcal{Y}$ be the set of all Young diagrams (or partitions) $Y=\left(n \geq \lambda_{1} \geq \lambda_{2} \geq\right.$ $\cdots \geq \lambda_{n}$ ) with at most $n$ rows (or parts) and with all rows of length at most $n$. That is, $\mathcal{Y}$ is the set of all Young diagrams that fit inside an $n \times n$ box. To a Young diagram $Y \in \mathcal{Y}$, we can associate an irreducible component $X_{Y}$ as in [1]. Such an irreducible component can be pictured as in Figure 2.

Proposition 9.1. The association $Y \leftrightarrow X_{Y}$ is a one-to-one correspondence between the set $\mathcal{Y}$ and the set of irreducible components $B_{Q}^{g}(\mathbf{w})$. Furthermore, each irreducible component is a point.

Proof. The case of type $A_{\infty}$ is done in [1, Thm 5.1]. The case of type $A_{2 n-1}$ is exactly analogous (see [1, Rem 5.4]). The fact that the irreducible components are points can either be seen directly or from the dimension formula for quiver varieties (see [13, Cor 3.12]).

For a Young diagram $Y$, let $\tilde{Y}$ denote the conjugate Young diagram. Thus if $Y=\left(\lambda_{1} \geq \cdots \geq \lambda_{m}\right)$ then $\tilde{Y}=\left(\mu_{1} \geq \cdots \geq \mu_{k}\right)$ where $\mu_{i}=\#\left\{j \mid \lambda_{j} \geq i\right\}$. Pictorially, $\tilde{Y}$ is obtained from $Y$ by reflection in the diagonal.

Proposition 9.2. We have $\mathbf{a}\left(X_{Y}\right)=X_{\tilde{Y}}$. 


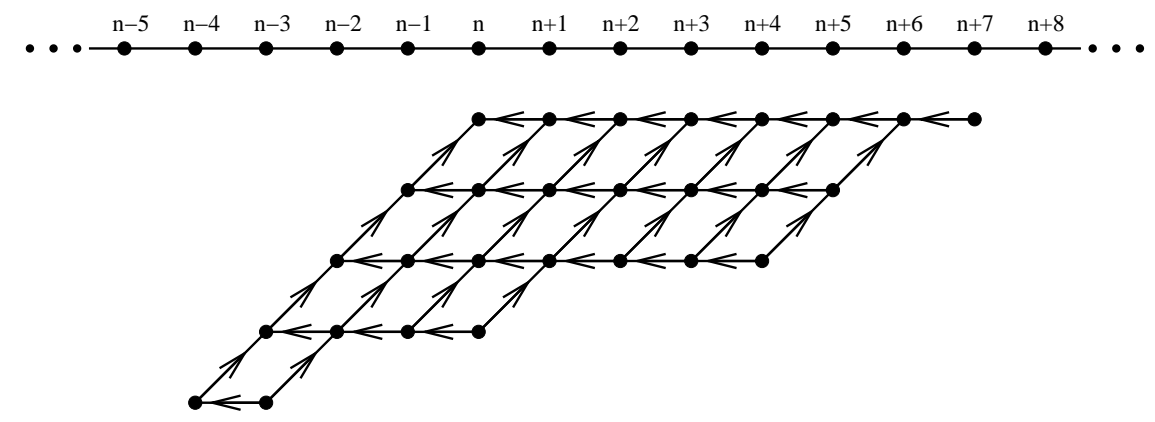

Figure 2. The irreducible component $X_{Y}$ corresponding to the Young diagram $(8,7,7,4,2)$. The Dynkin diagram is shown above the Young diagram. Each vertex in the Young diagram corresponds to a basis vector in the representation of the quiver. Its degree is given by the vertex of the Dynkin diagram above it. The arrows in the Young diagram indicate the action of $x$ (i.e. $x$ takes the vector at the tail of an arrow to the vector at the tip and all other components of $x$ are zero).

Proof. This is easy to see from our explicit description of the irreducible components $X_{Y}$. It also follows from dimension arguments since there is at most one irreducible component of a given dimension.

Let $\mathcal{Y}^{\mathrm{a}}$ be the set of self-conjugate elements of $\mathcal{Y}$. Then we have that

$$
B_{\Gamma}^{g}(\mathbf{w})=\left\{X_{Y} \mid Y \in \mathcal{Y}^{\mathbf{a}}\right\} .
$$

Now, as can be seen in Figure 2, the Young diagrams in $\mathcal{Y}$ can be thought of as consisting of vertices of various degrees (between 1 and $2 n-1$ ). For a Young diagram $Y \in \mathcal{Y}$, let $Y_{k}^{+}$(resp. $Y_{k}^{-}$) be the Young diagram in $\mathcal{Y}$ obtained from $Y$ by adding (resp. removing) a vertex of degree $k$ if such a Young diagram exists and let $Y_{k}^{ \pm}$be the ghost partition $\nabla$ if such a Young diagram does not exist. Using the fact that the quiver varieties are points, it is easy to see (see $[1,18]$ ) that the action of the Kashiwara operators $\tilde{e}_{k}$ and $\tilde{f}_{k}$ on $B_{Q}^{g}(\mathbf{w})$ is given by $\tilde{e}_{k}\left(X_{Y}\right)=X_{Y_{k}^{-}}$and $\tilde{f}_{k}\left(X_{Y}\right)=X_{Y_{k}^{+}}$where $X_{\nabla}=0$.

For $Y \in \mathcal{Y}$, let $Y_{i, j}^{++}$(resp. $Y_{i, j}^{--}$) be the Young diagram in $\mathcal{Y}$ obtained from $Y$ by adding (resp. removing) a vertex of degree $i$ and a vertex of degree $j$. Then we have the following theorem.

Theorem 9.3. The set $\mathcal{Y}^{\mathrm{a}}$ with operators

$$
\begin{gathered}
\tilde{e}_{k}(Y)=Y_{k, 2 n-k}^{--}, \quad \tilde{f}_{k}=Y_{k, 2 n-k}^{++}, \quad 1 \leq k \leq n-1, \\
\tilde{e}_{n}(Y)=Y_{n}^{-}, \quad \tilde{f}_{n}(Y)=Y_{n}^{+}, \\
\operatorname{wt}(Y)=\omega_{n}-\sum_{i=1}^{n} \#\{\text { vertices in } Y \text { of degree } i\} \alpha_{i}, \\
\varepsilon_{k}(Y)=\max \left\{k \mid, \tilde{e}_{k}(Y) \neq 0\right\}, \quad \varphi_{k}(Y)=\max \left\{k \mid \tilde{f}_{k}(Y) \neq 0\right\},
\end{gathered}
$$

where we identify the ghost partition $\nabla$ with zero, is isomorphic to the crystal of the spin representation of type $B_{n}$. 
Proof. This follows immediately from Proposition 7.4 and the above discussion. We have identified $Y \in \mathcal{Y}^{\mathrm{a}}$ with the irreducible component $X_{Y}$.

Proposition 9.4. Let $V$ be the vector space spanned by $\mathcal{Y}^{\mathbf{a}}$, let $e_{k}$ and $f_{k}$ act on this space by extending the action of $\tilde{e}_{k}$ and $\tilde{f}_{k}$ by linearity, and define $h_{k}(Y)=$ $\left\langle h_{k}, \operatorname{wt}(Y)\right\rangle Y$ (extended by linearity). Then $V \cong V\left(\omega_{n}\right)$ as representations of $\mathfrak{s o}_{2 n+1}$ where $\left\{e_{k}, f_{k}, h_{k}\right\}_{k=1}^{n}$ are the usual Chevalley generators. That is, we can realize the spin representation in a natural way on the space spanned by the selfconjugate Young diagrams fitting inside an $n \times n$ box.

Proof. This can be shown by direct computation. One merely checks that the necessary commutation relations hold.

This realization of the spin representation of type $B_{n}$ is similar to the realization of the spin representation of type $D_{n}$ on a certain set of Young diagrams obtained in $[18]$.

\section{REFERENCES}

[1] I. B. Frenkel and A. Savage. Bases of representations of type $A$ affine Lie algebras via quiver varieties and statistical mechanics. International Mathematics Research Notices, (28):1521$1548,2003$.

[2] I. Grojnowski and G. Lusztig. A comparison of bases of quantized enveloping algebras. In Linear algebraic groups and their representations (Los Angeles, CA, 1992), volume 153 of Contemp. Math., pages 11-19. Amer. Math. Soc., Providence, RI, 1993.

[3] J. Hong and S.-J. Kang. Introduction to quantum groups and crystal bases. American Mathematical Society, 2000.

[4] A. Hubery. Quiver representations respecting a quiver automorphism: a generalisation of a theorem of Kac. J. London Math. Soc. (2), 69(1):79-96, 2004.

[5] V. G. Kac. Infinite dimensional Lie algebras. Cambridge University Press, 1990.

[6] M. Kashiwara. On crystal bases of the $q$-analogue of universal enveloping algebras. Duke Math. J., 63:465-516, 1991.

[7] M. Kashiwara and Y. Saito. Geometric construction of crystal bases. Duke Mathematical Journal, 89(1):9-36, 1997.

[8] P. Littelmann. Characters of representations and paths in $\mathfrak{H}_{\mathbf{R}}^{*}$. In Representation theory and automorphic forms (Edinburgh, 1996), volume 61 of Proc. Sympos. Pure Math., pages 29-49. Amer. Math. Soc., Providence, RI, 1997.

[9] G. Lusztig. Canonical bases arising from quantized enveloping algebras. J. Amer. Math. Soc., 3(2):447-498, 1990.

[10] G. Lusztig. Quivers, perverse sheaves, and quantized enveloping algebras. Journal of the American Mathematical Society, (4):365-421, 1991.

[11] G. Lusztig. Introduction to Quantum Groups. Birkhäuser, Boston, 1993.

[12] H. Nakajima. Instantons on ALE spaces, quiver varieties, and Kac-Moody algebras. Duke Mathematical Journal, 76(2):365-416, 1994.

[13] H. Nakajima. Quiver varieties and Kac-Moody algebras. Duke Mathematical Journal, 91(3):515-560, 1998.

[14] M. Okado, A. Schilling, and M. Shimozono. Virtual crystals and fermionic formulas of type $D_{n+1}^{(2)}, A_{2 n}^{(2)}$, and $C_{n}^{(1)}$. Represent. Theory, 7:101-163 (electronic), 2003.

[15] M. Okado, A. Schilling, and M. Shimozono. Virtual crystals and Kleber's algorithm. Comm. Math. Phys., 238(1-2):187-209, 2003.

[16] Y. Saito. Crystal bases and quiver varieties. Mathematische Annalen, 324(4):675-688, 2002.

[17] A. Savage. Geometric and combinatorial realizations of crystal graphs. arXiv:math.RT/0310314.

[18] A. Savage. A geometric realization of spin representations and Young diagrams from quiver varieties. arXiv:math.AG/0307018.

[19] F. Xu. A note on quivers with symmetries. arXiv:q-alg/9707003. 
The Fields Institute for Research in Mathematical Sciences and University of Toronto, Toronto, Ontario, Canada

E-mail address: alistair.savage@aya.yale.edu 